\title{
Efficacy and Mechanism of the Combination of PARP and CDK4/6 Inhibitors in the Treatment of Triple-negative Breast Cancer
}

\section{XIUZHI ZHU}

Fudan University Shanghai Cancer Center

\section{CHEN}

Fudan University Shanghai Cancer Center

\section{Binhao Huang}

Fudan University Shanghai Cancer Center

\section{Xiaoguang Li}

Fudan University Shanghai Cancer Center

\section{Yang Liu}

Fudan University Shanghai Cancer Center

Xin $\mathrm{Hu}$

Fudan University Shanghai Cancer Center

\section{Zhimin Shao}

Fudan University Shanghai Cancer Center

zhonghua wang ( $\nabla$ wangzhonghua2691@sina.com )

Fudan University Shanghai Cancer Center

\section{Research}

Keywords: triple-negative breast cancer, PARP inhibitors, CDK4/6 inhibitors, Wnt signaling pathway, resistance

Posted Date: December 14th, 2020

DOl: https://doi.org/10.21203/rs.3.rs-125331/v1

License: (c) (i) This work is licensed under a Creative Commons Attribution 4.0 International License. Read Full License

Version of Record: A version of this preprint was published at Journal of Experimental \& Clinical Cancer Research on April 8th, 2021. See the published version at https://doi.org/10.1186/s13046-021-01930-w. 


\section{Abstract}

Background: PARP inhibitors (PARPi) benefit only a fraction of breast cancer patients with BRCA mutations and their efficacy is even more limited in triple-negative breast cancer (TNBC) due to clinical primary and acquired resistance. Here, we found that the efficacy of PARPi in TNBC can be improved with CDK4/6 inhibitors (CDK4/6i).

Methods: We screened primary PARPi-sensitive and resistant cell lines from existing BRCA ${ }^{\text {mut }}$ /TNBC cell lines and generated cells with acquired PARPi resistance by gradually increasing the concentration. The effects of the PARPi olaparib and the CDK4/6i palbociclib on BRCA ${ }^{\text {mut }} / T N B C$ cell lines were examined in both sensitive and resistant cells in vitro and in vivo. Pathway and gene alterations were assessed mechanistically and pharmacologically.

Results: We demonstrated for the first time that the combination of PARPi and CDK4/6i has synergistic effects against BRCA ${ }^{\text {mut }} / T N B C$ both in vitro and in vivo. In the PARPi-sensitive MB436 cells, the single agent olaparib significantly inhibited cell viability and affected cell growth due to severe DNA damage. In the PARPi-resistant HCC1937 and SUM149 cells, single-agent olaparib was ineffective due to potential homologous recombination (HR) repair, and the combination of PARPi and CDK4/6i greatly inhibited HR during the $\mathrm{G} 2$ phase, increased DNA damage and inhibited tumor growth. Inadequate DNA damage caused by PARPi activated the Wnt signaling pathway and upregulated MYC. Further experiments indicated that the overexpression of $\beta$-catenin, especially its hyperphosphorylation at the Ser675 site activated the Wnt signaling pathway and mediated PARPi resistance, which could be strongly inhibited by the combined treatment with CDK4/6i.

Conclusions: Our data provide a rationale for the clinical evaluation of the therapeutic synergy of PARPi and CDK4/6i in BRCA ${ }^{\text {mut }} /$ TNBCs with high Wnt signaling activation, high MYC expression and do not respond to PARPi monotherapy.

\section{Background}

Breast cancer (BC) is the most common malignancy and the leading cause of cancer-related death among women worldwide[1,2]. Triple-negative breast cancer (TNBC) accounts for approximately $15 \%$ of all BCs and lacks estrogen receptor (ER) and progesterone receptor (PR) expression and human epidermal growth factor receptor 2 (HER2) amplification[3]. Compared to other BC subtypes, TNBC has an inherently aggressive clinical behavior and does not respond to endocrine therapy or anti-HER2 targeted therapy. TNBC patients typically have a poorer outcome. For TNBC, chemotherapy is the primary established systemic treatment[4]. Currently, the clinical targeted drugs for BC include poly-(ADP)-ribose polymerase (PARP) inhibitors (PARPi), CDK4/ 6 inhibitors (CDK4/6i), PI3K inhibitors, and AKT inhibitors, but none of these drugs alone is very effective in TNBC. There is an urgent need to explore rational drug compatibility and potential targets for TNBC in the future[5,6]. 
PARPi are small molecule inhibitors of the PARP family of DNA repair enzymes[7]. Tumor cells that lack BRCA1 or BRCA2 are deficient in error-free homologous recombination (HR), and DNA double-stranded breaks (DSBs) accumulated during DNA replication need to be repaired by alternative, error-prone repair pathways, which results in incorrect repair of the DNA lesions caused by PARPi and causes cytotoxicity[8]. As a prime example of the concept of synthetic lethality in cancer, PARPi have achieved great successes in the treatment of BRCA1/2-mutated tumors, such as ovarian cancer[9] and BC[10]. In 2018, both PARPi olaparib and talazoparib were approved by the Food and Drug Administration (FDA) for the treatment of germline BRCA-mutated (BRCA ${ }^{\text {mut }}$ ) and HER2-negative metastatic BC. Unfortunately, PARPi resistance has proven to be a major problem in the clinical treatment of $B C[8]$, especially TNBC. In our clinical trial FUTURE, all three TNBC patients who had germline BRCA1/2 mutations were treated with PARPi and had progressed at the first evaluation[6]. Therefore, identifying who to treat, combating drug resistance and optimizing combination therapy will be the focus of research on PARPi[11]. Rational drug combinations that include PARPi may expand the patient population who may benefit from this drug class[12].

Cyclin and cyclin-dependent kinase (CDK) play an important role in the progression of the cell cycle. Among them, CDK4/ 6 activation regulates the transition of the cell cycle from $\mathrm{G} 1$ phase to $S$ phase[13]. CDK4/6i that arrest cells in $\mathrm{G} 1$ phase have been approved by the FDA for the treatment of ER-positive advanced $B C[14-17]$ and are expected to provide new treatment strategies for TNBC patients, especially the luminal androgen receptor (LAR) subtype of TNBC[18].

A recent study showed that CDK4/6i prevented recovery from multiple DNA-damaging agents[19]. Another study showed that the combination of CDK4/6i and PARPi might have a potential effect in ovarian cancer[20], but the specific mechanism remains unknown. In this article, we first test the hypothesis that the combination of CDK4/6i and PARPi synergistically inhibits the growth of TNBCs, thereby providing new therapeutic opportunities for certain TNBC populations.

\section{Materials And Methods}

\section{Cell culture and reagents}

Human BC MDA-MB-231, MDA-MB-468, CAL-148, MDA-MB-453, MDA-MB-157, MDA-MB-436, and HCC1937 cell lines were obtained from Nanjing CoBioer Biosciences (CoBioer, China) in 2018. The SUM149 cell line was obtained from the Cell Bank of the Chinese Academy of Sciences (Shanghai, China). The human embryonic kidney HEK293T cell line was kindly provided by Prof. Guo-Hong Hu (Shanghai Institutes for Biological Sciences) in 2014. All of the above cell lines were authenticated by DNA profiling (short tandem repeat, STR), morphology, cell viability, isoenzymes, and mycoplasma assays. MDA-MB-436 cells were maintained in Leibovitz's L15 medium (BasalMedia, L620) with $10 \mu \mathrm{g} / \mathrm{ml}$ insulin and $16 \mu \mathrm{g} / \mathrm{ml}$ glutathione, HCC1937 cells were maintained in RPMI 1640 medium (BasalMedia, L210), and SUM149 cells were maintained in DMEM/F12 (BasalMedia, L310) with $1 \mu \mathrm{g} / \mathrm{ml}$ hydrocortisone and $5 \mu \mathrm{g} / \mathrm{ml}$ insulin. The other cells were maintained in DMEM (BasalMedia, China, L110). 
All media were supplemented with $10 \%$ fetal bovine serum (Gibco, USA, 10270-106) and 1\% penicillinstreptomycin (BasalMedia, S110B), and the cells were not passaged more than six times from collection to use. Olaparib (AZD2281, S1060), veliparib (ABT-888, S1004), palbociclib (PD-0332991, S1579), and ribociclib (LEE011, S7440) were purchased from Selleck (USA). For the administration of various inhibitors, cells were treated with $10 \mu \mathrm{M} \mathrm{H-89}$ from Med Chem Express (MCE, USA, HY-15979) and $10 \mu \mathrm{M}$ MG-132 (MCE, HY-13259) as indicated.

Acquired olaparib-resistant cell lines were generated by culturing cells in media supplemented with increasing concentrations of olaparib for 8 months starting at $0.5 \mu \mathrm{M}$ and reaching a final dose of $20 \mu \mathrm{M}$. Resistant cells were maintained in media supplemented with $20 \mu \mathrm{M}$ olaparib.

\section{Cell viability assay and determination of drug synergy}

The cells of interest $\left(1 \times 10^{3} \sim 3 \times 10^{3}\right.$ cells per well) were seeded into 96 -well plates in triplicate overnight in $100 \mathrm{ml}$ of complete growth medium and then treated with the indicated drugs for the designed time. Cell viability was tested using the cell counting kit-8 (CCK-8) assay (Dojindo Molecular Technologies, Japan, CK04) as the manufacturer's instructions. The combination index ( $\mathrm{Cl})$ was used to evaluate the synergistic effect of the two drugs and was calculated by the Chou-Talalay method with CompuSyn software[21].

\section{Flow cytometry analysis}

Flow cytometry analysis was used to assess cell apoptosis and the cell cycle. The cells of interest were treated with the designed drugs for 3 days and digested with EDTA-free trypsin. For apoptosis evaluation, the cells were collected and stained using an Annexin V-FITC/PI apoptosis kit (MultiSciences, Hangzhou, China, AP101). For cell cycle analysis, the cells were fixed using $70 \%$ precooled ethanol at $4^{\circ} \mathrm{C}$ overnight, washed with PBS and stained with PI solution (MultiSciences, AP101-60-PI). The above cells were all identified and quantified by a flow cytometer (Beckman Cytomics FC 500 BD FACSCanto II) according to the manufacturer's instructions, and the data were analyzed by FlowJo v10 software.

\section{Migration assay}

The cells used for the Transwell (Corning, USA) migration assay were pretreated with specific drugs for 3 days. Then, a total of $5 \sim 10 \times 10^{4}$ cells were resuspended in the upper compartment of each chamber with $100 \mu \mathrm{l}$ serum-free medium, and the lower chamber was filled with $600 \mu \mathrm{l}$ medium containing $20 \%$ fetal bovine serum. The Transwell plates were incubated in a humidified environment with $95 \%$ air and $5 \% \mathrm{CO}_{2}$ at $37^{\circ} \mathrm{C}$ for $18 \mathrm{~h}$ (HCC1937) or $24 \mathrm{~h}$ (MDA-MB-436). The chambers were washed with PBS, fixed with formaldehyde and stained with $0.5 \%$ crystal violet. The migrated cells were imaged and counted using ImageJ (National Institutes of Health, Bethesda, MD, USA).

\section{Senescence assay}


Following the manufacturer's protocol, a $\beta$-Galactosidase Staining Kit (Solarbio, Beijing, China, G1580) was used for the detection of cell senescence according to Dimri et al[22]. Images were taken in transmitted light by a computerized imaging system consisting of a Leica charge-coupled device (CCD) DFC420 camera and a Leica DM IRE2 microscope (Leica Microsystems Imaging Solutions Ltd).

\section{Colony formation assay}

For the colony formation assay, 500 cells were plated into 6-well or 12-well plates. After overnight incubation, the cells were treated with the designed drugs for 12-17 days, and the medium was replaced every 3 days. The colonies were fixed in formaldehyde and stained with $0.5 \%$ crystal violet. Colonies consisting of 50 or more cells were included in the count.

\section{Immunofluorescent staining}

Immunofluorescent staining was carried out as described previously[23,24]. Briefly, after drug treatment, the adherent cells were washed in PBS, fixed in $4 \%$ paraformaldehyde, permeabilized in $0.1 \%$ Triton X-100,

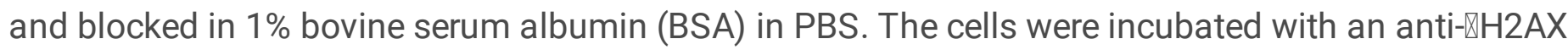

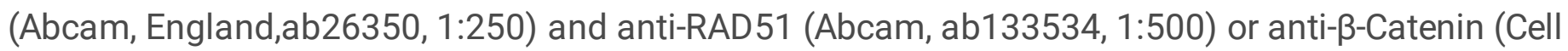
Signaling Technology, USA, D10A8 \#8480, 1:100) antibody in $1 \%$ BSA at $4^{\circ} \mathrm{C}$ overnight, washed three times in PBS for five minutes each, and then incubated with the appropriate secondary antibody (Jackson ImmunoResearch, USA, 115-095-003/111-585-003). DNA staining was performed using Gold Antifade Mountant with DAPI (Invitrogen, USA, P36931). Leica SP5 confocal laser scanning microscopy (Leica Microsystems, Buffalo Grove, USA) was used for immunofluorescence imaging.

\section{HR assay}

The HR assay has been described in previous research[25,26]. In brief, a clone stably expressing pDR-GFP was generated and validated by analyzing GFP-positive cells. For HR assays, cells stably expressing DRGFP were transfected with IScel-GR plasmids. Twenty-four hours post transfection, the cells were treated with $10 \mu \mathrm{mol} / \mathrm{L}$ triamcinolone acetonide (TA) and cultured for another $48 \mathrm{~h}$ [27]. To study the effect of drugs on HR, we added a specific concentration of drugs together with TA. The proportion of GFP-positive cells was evaluated using flow cytometry, and the efficiency of HR was calculated.

\section{RNA-Seq analysis}

Total RNA from MDA-MB-436 and HCC1937 cells treated with DMSO, $5 \mu \mathrm{M}$ olaparib, $5 \mu \mathrm{M}$ palbociclib, or their combination (Ola/Palb) for $24 \mathrm{~h}$ was extracted using TRIzol Reagent (Invitrogen). RNA quantity and quality were assessed by an Agilent 2100 Bioanalyzer (Agilent Technologies, Palo Alto, CA, USA), NanoDrop (Thermo Fisher Scientific Inc.) and 1\% agarose gel. One microgram of total RNA with an RNA integrity number (RIN) value above 6.5 was used for cDNA library construction. The cDNA libraries created from the cell lines (sixteen samples in total, with duplicate libraries for each sample) were run on a HiSeq 2500 instrument according to the manufacturer's instructions (Illumina, San Diego, CA, USA). 
Gene set enrichment analysis (GSEA) was performed by the JAVA program using MSigDB Hallmark gene set collection. One thousand random sample permutations were carried out, and the significance threshold was set at NES absolute value $>1$, NOM $p$-value $<0.05$ and FDR q-value $<0.05$.

\section{Real-time quantitative reverse transcription PCR (qRT-PCR)}

Total RNA was isolated from specimens or cells using the RNeasy Plus Mini Kit (QIAGEN, Germany) following the manufacturer's protocol. cDNA was synthesized using a PrimeScript RT Reagent Kit with gDNA Eraser (TaKaRa, Japen, RR047A). Real-time qRT-PCR was carried out using SYBR Premix Ex Taq (TaKaRa, RR420A) in triplicate on an ABI 7900HT Fast Real-Time PCR System (Applied Biosystems). All primers were synthesized by Sangon Biotech, and the sequences of the primers are available in

Supplementary Table S1. The results were analyzed with SDS v2.1 software and the $2^{-\triangle \Delta C T}$ method by normalization to GAPDH levels.

\section{Western blot (WB) analysis}

The WB protocol has been described in detail previously[28]. In short, cells were lysed in Pierce T-PER® Tissue Protein Extraction Reagent (Thermo Fisher Scientific Inc.) containing protease and phosphatase inhibitors (Bimake, USA, B14001, B15001A+B). The lysates were centrifuged at $12000 \mathrm{rpm}$ for $15 \mathrm{~min}$, the supernatants were collected, and protein concentrations were determined with a bicinchoninic (BCA) protein assay kit (Solarbio, PC0020). A total of $20-30 \mu \mathrm{g}$ of protein was separated by SDS-PAGE and transferred to PVDF membranes (Millipore, USA, IPVH00010, ISEQ00010). The primary and secondary antibodies are described in Supplementary Table S2. For the immunoprecipitation (IP) analysis, the cells were lysed in NP-40 lysis buffer (Beyotime, China, P0013F) containing protease and phosphatase inhibitors. The supernatants were incubated with 1-3 $\mu$ g primary antibodies overnight at $4^{\circ} \mathrm{C}$ on a rotating platform, followed by immunoblotting analysis. ImageJ was used to quantify the immunoblotting results by measuring the protein band densities.

\section{Expression vectors, plasmid transfection and lentiviral infection}

The CTNNB1 (NM_001904) and MYC (NM_002467) cDNAs were obtained from GeneChem (Shanghai, China) and subcloned into the Ubi-MCS-3FLAG-CBh-gcGFP-IRES-puromycin vector (GV492) to generate the Flag-gcGFP-CTNNB1 and Flag-gcGFP-MYC expression vectors, respectively. Site-directed mutations CTNNB1S675A and CTNNB1S675D were generated by PCR-based mutagenesis and verified by DNA sequencing. Human CTNNB1 short hairpin RNAs (shRNAs) in the U6-MCS-Ubiquitin-Cherry-IRESpuromycin vector (GV298) and MYC shRNAs in the U6-MCS-Ubiquitin-EGFP-IRES-puromycin vector (GV248) were purchased from GeneChem. Detailed information concerning DNA constructs and the primers used for molecular cloning is provided in Supplementary Table S1. To generate stable cell lines expressing cDNAs or shRNAs, each lentiviral expression vector was transfected into HEK293T cells with polyethyleneimine (PEI). The supernatant containing viruses was collected $48 \mathrm{~h}$ after transfection, filtered, and used to infect target cells in the presence of $10 \mu \mathrm{g} / \mathrm{ml}$ polybrene (Sigma-Aldrich, USA, H9268) prior to 
drug selection with 1-2 $\mu \mathrm{g} / \mathrm{ml}$ puromycin for one week. Overexpression (OE) and knockdown (KD) efficiencies were validated by immunoblotting after transfection.

The lentiCas9-Blast (Addgene, USA, \#52962) and lentiGuide-Puro (Addgene, \#52963) vectors were provided by the Feng Zhang laboratory. The MYC knockout (KO) cell line was generated using the CRISPR/Cas 9 system[29] and was validated by Sanger sequencing and immunoblotting analysis. The individual sgRNA sequences are provided in Supplementary Table S3.

\section{Xenograft in vivo model}

All animal experiments were performed in compliance with the NIH Guide for the Care and Use of Laboratory Animals (http://oacu.od.nih.gov/regs/index.htm) and were approved by the Fudan Animal Ethics Committee (approval number, 201911004S). MDA-MB-436 and HCC1937 cells ( $8 \times 10^{6}$ per mouse) were harvested and resuspended in $50 \mu \mathrm{L}$ of PBS and $50 \mu \mathrm{L}$ of Corning ${ }^{\circledR}$ Matrigel ${ }^{\circledR}$ (BD Biocoat, USA, 354248). Then the cells were injected directly into the mammary fat pads of six-week-old female NODSCID mice weighing 15 to $16 \mathrm{~g}$. Tumor volumes were monitored via caliper measurements every 3-4 days and were calculated as follows: length $\times$ width ${ }^{2} / 2$. When tumors reached approximately $75 \mathrm{~mm}^{3}$, the mice were randomly allocated into four groups administered control solvent, olaparib, palbociclib, or their combination (Ola/Palb) once a day. Olaparib was dissolved in $4 \% \mathrm{DMSO}+30 \% \mathrm{PEG} 300+\mathrm{ddH}_{2} \mathrm{O}$ for intraperitoneal administration and dosed at $50 \mathrm{mg} / \mathrm{kg} /$ day; palbociclib was dissolved in $0.9 \%$ warm saline and administered via oral gavage at $100 \mathrm{mg} / \mathrm{kg} /$ day. After 21 days, the tumors were collected.

\section{Immunohistochemistry (IHC) analysis}

Details on the IHC protocol have been described previously [30-32]. For hematoxylin and eosin (H\&E) staining, slides were stained with Mayer's hematoxylin (Sigma-Aldrich) and $0.1 \%$ sodium bicarbonate and were counterstained with Eosin Y solution (Sigma-Aldrich). For IHC, the primary and peroxidase (HRP)conjugated secondary antibodies were listed in Supplementary Table S2. The positive-staining density was measured by the computerized imaging system mentioned above.

\section{Statistical analysis}

Quantification and statistical analysis were performed with GraphPad Prism 7.0 (GraphPad Software, Inc.), SPSS version 22.0 (SPSS, Chicago, IL) or R software version 3.5.3. utilizing the statistical tests described in the text and figure legends. The unpaired two tailed Student's $t$ test was used to compare data between two groups, and correlation coefficients were calculated using the Pearson test. All p-values were two-sided, and p-values less than 0.05 were considered statistically significant.

\section{Results}

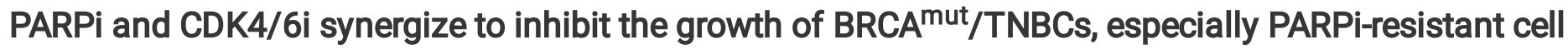
lines 
To evaluate the effects of the concomitant inhibition of PARP and CDK4/6, we assessed the response of a panel of 8 TNBC cell lines, including 3 LAR subtype cell lines and 3 BRCA ${ }^{\text {mut }}$ cell lines, to olaparib and palbociclib as single agents or in combination. Following a 5-day drug treatment, the half-maximal inhibitory concentration (IC50) of the two drugs was determined by the CCK-8 assay. Consistent with previous literature, the IC50 of the CDK4/6i palbociclib in the LAR subtype was lower than that in other TNBC subtypes, and the IC50 of the PARPi olaparib in most BRCA ${ }^{\text {mut }}$ cells was lower than that in wildtype cells (Fig. 1a). Among them, the IC50 of olaparib in PARPi-resistant BRCA ${ }^{\text {mut }}$ cell lines (HCC1937 and SUM149) was much greater than that in the BRCA ${ }^{\text {mut }}$ MDA-MB-436 (MB436) cells, which are considered to be sensitive to PARPi. Interestingly, palbociclib reduced the IC50 of olaparib in PARPi-resistant BRCAmut cell models but not in other cell lines (Fig. 1a).

Next, the PARPi-resistant HCC1937 cells and the sensitive MB436 cells were selected for follow-up experiments. The CompuSyn model was used to evaluate the synergistic effects of palbociclib and olaparib in the two cell lines. With the increased concentrations of PARPi and CDK4/6i, the growth inhibition of MB436 and HCC1937 cells became stronger, and the Cls were $0.72 \pm 0.25$ and $0.80 \pm 0.13$, respectively, suggesting the potential synergistic effect of the inhibitors (Fig. 1b). According to the $\mathrm{Cl}$ listed in Fig. S1a, the optimal drug concentration ratio (CR=olaparib/palbociclib) of PARPi to CDK4/6i was less than 1 in MB436 cells (CR<1), and it was probably greater than or equal to 1 in HCC1937 cells $(\mathrm{CR} \geq 1)$.

In the PARPi-sensitive MB436 cells, the single agent olaparib significantly inhibited cell viability and affected cell growth, while in the PARPi-resistant HCC1937 cells, only the combination treatment had a significant inhibitory effect (Fig. 1c and 1d). Under the combined treatment, reduced "synapse" between the cells and nuclear shrinkage were observed. In addition, olaparib combined with palbociclib inhibited cell migration (Fig. 1e) and promoted BRCA ${ }^{\text {mut }} /$ TNBC apoptosis (Fig. 1f) and cell senescence (Fig. S1b) more significantly than either agent alone in both MB436 and HCC1937 cells. To exclude the special effects of these two drugs, we tested the cell viability effect of two other drugs, the CDK4/6i ribociclib and PARPi veliparib, and consistent experimental results were obtained (Fig. S1c and S1d). We found that CDK4/6i resensitized the PARPi-resistant BRCA ${ }^{\text {mut }}$ cells to PARPi.

\section{CDK4/6i together with PARPi is synthetically lethal by inducing HR repair deficiency}

The synergistic activity of PARPi and CDK4/6i in the BRCA ${ }^{\text {mut } / T N B C}$ cells prompted us to examine whether they induced synthetic lethality through DNA damage repair or even HR repair mechanisms. Immunofluorescence staining analysis revealed that in the PARPi-sensitive MB436 cells, treating with olaparib alone showed a significant increase in the intensity of $\mathrm{yH} 2 \mathrm{AX}$ nuclear foci (a surrogate marker of DNA DSBs), while RAD51 foci (a marker of the competency of HR repair) were hardly observed (Fig. $\mathbf{2 b}$ ). In contrast, there was a significant increase in the intensity of RAD51 foci but nonsignificant changes in the intensity of $\mathrm{YH} 2 \mathrm{AX}$ foci after single olaparib treatment in the PARPi-resistant HCC1937 cells, indicating the potential HR repair ability of these cells and the protection of DNA from serious damage (Fig. 2a). Although the combination of olaparib and palbociclib in HCC1937 cells caused a short-term 
increase in RAD51 nuclear foci, the staining decreased rapidly after one day of treatment and was maintained over time, while $\mathrm{YH} 2 \mathrm{AX}$ foci cumulatively increased over time, indicative of persistent and severe DNA damage (Fig. 2b). The real-time qRT-PCR results also verified that in the PARPi-resistant model (HCC1937 and SUM149 cells), RAD51 increased under the single-agent treatment but decreased under the combined treatment; in the PARPi-sensitive model (MB436 cells), olaparib alone could maintain RAD51 at a low level (Fig. 2c) and cause DNA damage.

To confirm the effect of the CDK4/6i and PARPi combination on DNA repair, we next directly measured DNA HR repair by using specific fluorescent reporter assays[26,33]. As shown in Fig. 2d, compared with the vehicle, olaparib and palbociclib alone could reduce the level of HR repair, but HR repair was impaired more significantly in the combination treatment. These results indicated that CDK4/6 inhibition may induce deficiency in the HR repair of DSBs, which was a potential mechanism underlying the synthetic lethality of PARP inhibition.

\section{CDK4/6i results in defective $\mathrm{G} 2$ cell cycle-dependent DNA repair after chromosomal damage caused by PARPi}

Since palbociclib and olaparib act in different phases of the cell cycle, we next analyzed the effect of their combination on cell cycle progression by DNA content measurements. In PARPi-sensitive and resistant cells, the drugs had different effects on cell cycle progression (Fig. 2e). Cell cycle analysis following olaparib exposure showed an extended G2 phase and demonstrated the absence of G1 arrest $(p<0.05)$ after palbociclib exposure in HCC1937 and SUM149 cells, which was not observed in PARPi-sensitive MB436 cells. Moreover, compared to the extended G2 phase in response to the single PARPi, the G2 phase was greatly reduced following the combined treatment. These changes in cell cycle progression might be partly due to the $\mathrm{G} 1$ arrest of the cell cycle caused by CDK4/6i. We speculated that the reduction in some cell cycle-dependent DNA damage repairs, such as those in G2 phase, may cause irreversible lethal DNA damage under the combination of CDK4/6i and PARPi.

Since concomitant treatment with CDK4/6i and PARPi might affect the efficacy of the combined treatment, we evaluated the effect of the sequential administration of these drugs. We found that the combination treatment significantly inhibited colony formation in HCC1937 cells (Fig. 2f), whereas a 6day pretreatment with palbociclib (P-O, Fig. $\mathbf{2 f}$ and S2a) reduced the antiproliferative effect of olaparib. We observed a significant decrease in colony formation in the two resistant cell lines after a 6-day pretreatment with olaparib before the continuous administration of palbociclib (O-P). Similar data were obtained with ribociclib (Fig. S2b) in the PARPi-resistant cell lines. Regarding the relationship between cell cycle arrest and DNA damage, we speculated that CDK4/6i interferes with the cytotoxic effect of DNAdamaging agents, as CDK4/6i arrests cells in G1, thereby protecting the cells from damage during DNA replication or mitosis, which was consistent with some previous studies[34,35]. Under the combined treatment, the antitumor effect of CDK4/6i (inhibiting DNA repair and increasing DNA damage) may partly depend on the pretreatment effect of PARPi. 
We next explored in depth the molecular mechanism of the combination of PARPi and CDK4/6i. RNA sequencing analysis was applied in the PARPi-sensitive MB436 cells and the PARPi-resistant HCC1937 cells, and the cells were treated with vehicle, olaparib, palbociclib or a combination of the two for $24 \mathrm{~h}$ due to significantly high expression of c-PARP and downregulation of pRB (S807/811) in $24 \mathrm{~h}$ (Fig. S3a). GSEA revealed that MYC target gene sets were significantly downregulated, while immune-related pathways (alpha/gamma interferon response) were significantly upregulated in MB436 cells treated with olaparib (Fig. 3a and 3c). In PARPi-resistant BRCA mut HCC1937 cells, many MYC target genes were strongly downregulated only upon combined drug treatments, but the changes were not significant with olaparib alone (NES=-1.17, NOM p-value=0.139, FDR q-value=0.135) (Fig. $3 \mathrm{~b}$ and $3 \mathrm{c}$ ). In addition, in HCC1937 cells, DNA replication and DNA damage repair gene sets (mitotic spindle and UV response DN) were upregulated after single-agent olaparib treatment (Fig. S3b). More interestingly, the combined treatment could downregulate the gene sets that were upregulated by the single agent olaparib in PARPiresistant cell lines, such as the Wnt beta catenin signaling (Fig. S3c), G2/M checkpoint and E2F target genes (Fig. 3d). These pathways might play an important role in PARPi resistance and to some extent explain the important mechanism by which CDK4/6i resensitizes PARPi-resistant cells.

To clarify the relationship between the Wnt pathway and MYC, we then selected $\beta$-catenin (the core molecules in the Wnt pathway) and c-myc for WB analysis in HCC1937 and SUM149 cell lines and found that the Wnt pathway acts upstream to regulate the expression of c-myc (Fig. 3e and 3f, Fig. S3d and S3e), which was consistent with previous studies[36,37]. Subsequent qRT-PCR and WB analyses confirmed that treatment with olaparib alone for $24 \mathrm{~h}$ in the PARPi-sensitive MB436 cells resulted in significant downregulation of Wnt beta catenin signaling (CTNNB1, TCF4, Axin2, C-Jun and Cyclin D1) and MYC; in the PARPi-resistant HCC1937 and SUM149 cells, olaparib alone resulted in remarkable upregulation of these genes, which were downregulated by the combined treatment with olaparib and palbociclib (Fig. $3 g$ and $3 h$ ).

The entry of $\beta$-catenin into the nucleus plays a central role in the activation of the classical Wnt pathway. In-depth WB analysis revealed that the nucleoplasmic distribution of $\beta$-catenin changed before an obvious change was found in the total level of catenin protein (Fig. S3f and Fig 3i). The 12-h combined treatment prevented $\beta$-catenin from entering the nucleus in HCC1937 cells (Fig. 3I) and reduced the expression of MYC, although the total amount of $\beta$-catenin was not significantly reduced. Moreover, the expression of MYC was positively correlated with the level of $\beta$-catenin in the nucleus, and the effects were both time- and dose-dependent (Fig. 3j and 3k).

Previous studies have shown that DNA damage can activate the Wnt pathway. We therefore assumed that DNA damage caused by PARPi activated the Wnt signaling pathway and upregulated MYC, leading to resistance to PARPi. Overall, the combined use of PARPi and CDK4/6i inhibits MYC expression through the Wnt pathway.

Ser675 phosphorylation of $\beta$-catenin in the Wnt signaling pathway mediates resistance to PARPi 
The phosphorylation of different sites of $\beta$-catenin affects its nuclear accumulation, protein stability and signaling activity[38-40]. Phosphorylation at Ser33, Ser37, Thr41 or Ser45 promotes $\beta$-catenin degradation and inhibits the classical Wnt pathway, while phosphorylation at Ser552 or Ser675 stabilizes $\beta$-catenin and promotes its nuclear translocation, which positively regulates the Wnt signaling pathway. We next tested the phosphorylation levels of different sites of $\beta$-catenin under different drug treatments for $12 \mathrm{~h}$. We were surprised to find that the combination treatment of olaparib and palbociclib greatly reduced phosphorylation at Ser675 in the nucleus (Fig. 4a). Compared to those in the PARPi-sensitive BRCA $^{\text {mut }}$ TNBC MB436 cell line, the Ser552 and Ser675 of $\beta$-catenin in the PARPi-resistant HCC1937 and SUM149 cells both showed higher phosphorylation, and Rad51 and $\beta$-catenin were also highly expressed (Fig. 4b). In contrast, the phosphorylation sites (Ser33, Ser37, Thr41 and Ser45) that negatively regulated the Wnt signaling pathway were highly phosphorylated in MB436 cells (Fig. S3g).

Cells with acquired olaparib resistance (HCC1937) were generated by treatment with olaparib in a stepwise dose-escalating fashion for 8 months and were maintained in media supplemented with $20 \mu \mathrm{M}$ olaparib. The resistant cells were cross resistant to another PARPi, veliparib (Fig. S4). The CCK-8 assay verified that the IC50 of olaparib in the acquired olaparib-resistant HCC1937 cells was nearly three times that of the parent cells (Fig. 4c). Compared to the parental cell lines, acquired-resistant HCC1937 cells expressed high levels of $\beta$-catenin, $c$-myc and Rad51, and the Ser675 site of $\beta$-catenin was also highly phosphorylated, which was positively correlated with Wnt signaling pathway activation (Fig. 4d). Notably, CTNNB1 overexpression in parent cells confirmed by WB analysis (Fig. 3e) also resulted in drug resistance to olaparib (Fig. 4e).

We next generated two $\beta$-catenin mutants S675D (Ser-to-Asp) and S675A (Ser-to-Ala) in the C-terminal domain in $\mathrm{HCC} 1937$ cells (Fig. 4f). Mutant $\beta$-catenin ${ }^{\text {S675D }}$ was an activating mutation that maintained continuous phosphorylation at site 675 , while mutant $\beta$-catenin ${ }^{5675 A}$ was an inhibitory mutation. Cells with $\beta$-catenin ${ }^{W T}$ or mutant $\beta$-catenin ${ }^{\mathrm{S} 675 \mathrm{D}}$ both showed resistance to high concentrations of olaparib, while cells with mutant $\beta$-catenin ${ }^{\mathrm{S} 675 \mathrm{~A}}$ were much more sensitive to olaparib (Fig. $\mathbf{4 g}$ ). Therefore, we hypothesized that the overexpression of $\beta$-catenin, especially its hyperphosphorylation at the Ser675 site activated the Wnt signaling pathway, thereby mediating PARPi resistance.

We also found that $\beta$-catenin and c-myc interacted with each other, which could protect c-myc from degradation and increase its stability (Fig. $4 \mathrm{~h}$ and $4 \mathrm{i}$ ). The phosphorylation of Ser675 affected the interaction of the two proteins (Fig. 4j).

\section{CDK4/6i can strongly inhibit the viability of PARPi-resistant cells}

In both HCC1937 cells with acquired resistance to olaparib and cells with high expression of $\beta$-catenin WT or mutant $\beta$-catenin ${ }^{5675 D}$, continuous activation of the Wnt signaling pathway and high expression of MYC made high concentrations of olaparib ineffective. Interestingly, a small dose of palbociclib greatly inhibited the viability of these cells (Fig. $\mathbf{4 e}$ and $\mathbf{4 g}$ ). In addition, the combined treatment had a stronger inhibitory effect in cells with $\beta$-catenin ${ }^{W T}$ or mutant $\beta$-catenin ${ }^{5675 D}$ compared with the control cell line (Fig. 
4e) and made no significant difference in the viability of cells with mutant $\beta$-catenin ${ }^{\text {S675D }}$ or $\beta$ catenin $^{\mathrm{S} 675 \mathrm{~A}}$.

\section{The PARPi and CDK4/6i synergically inhibited tumor growth in vivo}

We next evaluated the efficacy of the combination of olaparib and palbociclib in a xenograft mouse model of MB436 and HCC1937 cells. Consistent with the experiments in vitro, single-agent olaparib significantly inhibited the growth of the MB436 tumor model; however, the single agent had limited activity in the HCC1937 tumor model (Fig. 5a and 5b). The tumor growth in both models was significantly slowed under the combination treatment of olaparib and palbociclib.

As determined by histological analysis, the combination treatment resulted in a substantial decrease in $\mathrm{Ki}-67$ (a proliferation marker) and $\beta$-catenin but a significant increase in the formation of $\mathrm{YH} 2 \mathrm{AX}$ nuclear foci (Fig. 5c and 5d). Decreased proliferation and increased DNA damage may, at least in part, explain the observed response to the combined treatment. Consistent with the conclusions drawn in vitro, palbociclib alone or in combination with olaparib sufficiently suppressed $\beta$-catenin expression and inhibited the Wnt signaling pathway.

\section{Discussion}

The development of PARPi resistance in BRCA ${ }^{\text {mut }}$ cancers is a pressing clinical problem. Currently, resistance to PARPi can be classified into four main mechanisms: altered drug availability, affected (de)PARylation enzymes, restored HR, and restored replication fork stability[41]. To overcome the resistance to PARPi and enhance their efficacy, an increasing number of studies are currently exploring treatment strategies that can be combined with PARPi, including oncolytic herpes simplex viruses (oHSVs)[42,43], ionizing radiation[44], CDK inhibitors (CDK12i[45], CDK1/2i[46], CDK18i[47], etc.), immunotherapy[48,49] and epigenetic drugs (HDAC inhibitor[50] and DNMT inhibitor[51,52]).

Our study is the first to test the hypothesis that the combination of CDK4/6i and PARPi synergistically inhibits the growth of BRCA ${ }^{\text {mut }}$ TNBCs, thereby providing new treatment opportunities for certain TNBCs, especially those resistant to PARPi. We selected one PARPi-sensitive (MB436) and two relatively PARPiresistant BRCA ${ }^{\text {mut} / T N B C ~ c e l l ~ l i n e s ~(H C C 1937 ~ a n d ~ S U M 149) ~ o u t ~ o f ~} 8$ TNBC cell lines based on their response to PARPi treatment. In the PARPi-sensitive cells, the single agent olaparib significantly inhibited cell viability and affected cell growth in vitro and in vivo due to severe DNA damage. In the PARPiresistant cells, potential HR repair ability and protection of DNA from serious damage were observed after treatment with the single agent olaparib, making it ineffective. Only the combination with palbociclib could greatly inhibit HR and strongly suppress tumor growth.

In addition to the changes in HR and DNA damage that we found, the cell cycle distribution also changed with PARPi or CDK4/6i administration. Compared to the extended $G 2$ phase in response to treatment with PARPi alone in the PARPi-resistant BRCA ${ }^{\text {mut }} /$ TNBCs, the $G 2$ phase and G2/M checkpoint-related gene 
sets were greatly reduced following the combined treatment. A previous study showed that BRCA1 loss resulted in an accumulation of G1 DNA damage[53]. In the S and G2 phases, DNA replication occurs, sister chromatids are present, and cell DNA damage can be repaired by HR[41]. We speculate that the G1 arrest of the cell cycle caused by CDK4/6i increases the DNA damage in $\mathrm{G} 1$ phase caused by PARPi and BRCA1/2 mutations. In addition, the $\mathrm{G} 1$ phase arrest indirectly reduces $\mathrm{G} 2$ phase and $\mathrm{G} 2$-dependent HR. Upon the sequential administration of the two drugs, we found that PARPi followed by CDK4/6i had a better inhibitory effect. The antitumor effect of CDK4/6i in the combination treatment may partly depend on the pretreatment effect of PARPi that causes DNA damage and help PARPi increase DNA damage.

When exploring the molecular mechanism of the combination treatment in depth, MYC and the Wnt signaling pathway attracted our attention and were observed to mediate resistance to PARPi mainly based on the following reasons. First, the Wnt pathway was upregulated under PARPi-alone treatment and downregulated under the combination treatment in the PARPi-resistant cells. This may be consistent with the view that inadequate DNA damage can activate the Wnt signaling pathway[54]. Second, in PARPi-resistant cells, many MYC targets were strongly downregulated only upon combined drug treatments, while in the PARPi-sensitive cells, olaparib alone was sufficient. Third, the Wnt pathway acts upstream to regulate the expression of MYC. The role of MYC in the DNA damage response and genomic instability has been the subject of debate and controversy[55]. Previous studies have reported that deregulation of MYC could induce DNA damage and genomic instability[56,57]. We predicted that inadequate DNA damage caused by PARPi activated the Wnt signaling pathway and upregulated MYC, leading to resistance to PARPi. In addition, we also found activation of the Wnt signaling pathway in cells with acquired Olaparib-resistance. In follow-up studies, we found that any activation of the Wnt signaling pathway, such as the overexpression of $\beta$-catenin (the core molecule in the Wnt pathway) or its hyperphosphorylation at the Ser675 site (promoting its nuclear translocation, thereby positively regulating Wnt), could mediate resistance to PARPi but be inhibited by a small dose of the CDK4/6i palbociclib. Our studies could pave the way for novel treatment options to target primary and acquired PARPi resistance.

Our research also has some limitations. We did not explore the role of human immunity in drug resistance and resensitization. In addition, we did not study a clinical cohort to verify our hypothesis, and the existing database lacks medication information and cannot be used for verification.

\section{Conclusions}

to a certain extent, our data provide a rationale for the clinical evaluation of the therapeutic synergy of PARPi and CDK4/6i in BRCA ${ }^{\text {mut }} /$ TNBCs that show high Wnt signaling pathway activation, high MYC expression and do not respond to PARPi monotherapies.

\section{Abbreviations}

BC: Breast cancer; TNBC: Triple-negative breast cancer; ER: Estrogen receptor; PR: Progesterone receptor; HER2: Human epidermal growth factor receptor 2; PARP: Poly-(ADP)-ribose polymerase; PARPi : PARP 
inhibitors; CDK: Cyclin-dependent kinase; CDK4/6i: CDK4/6 inhibitors; HR: Homologous recombination; DSBs: DNA double-stranded breaks; FDA: Food and Drug Administration; LAR: Luminal androgen receptor; CCK-8: Cell counting kit-8; Cl: Combination index; CCD: Charge-coupled device; BSA: Bovine serum albumin; TA: Triamcinolone acetonide

RIN: RNA integrity number; GSEA: Gene set enrichment analysis; GEO: Gene Expression Omnibus; PCR: Polymerase chain reaction; qRT-PCR: quantitative reverse transcription PCR; WB: Western blot; BCA: Bicinchoninic acid; IP: Immunoprecipitation; shRNA: Short hairpin RNA; PEI: Polyethyleneimine; OE: Overexpression; KD: Knockdown; KO: Knockout; IHC: Immunohistochemistry; CR: Concentration ratio; oHSVs: Oncolytic herpes simplex viruses.

\section{Declarations}

\section{Ethics approval and consent to participate}

All in vivo animal experiments were performed in compliance with the NIH Guide for the Care and Use of Laboratory Animals (http://oacu.od.nih.gov/regs/index.htm) and were approved by the Fudan Animal Ethics Committee. All surgical procedures were

conducted under anaesthesia to minimise animal suffering.

\section{Consent for publication}

All authors agree to the publication of our work entitled "Efficacy and mechanism of the combination of PARP and CDK4/ 6 inhibitors in the treatment of triple-negative breast cancer" to Journal of Experimental \& Clinical Cancer Research.

\section{Availability of data and materials}

Please contact the corresponding author (Zhonghua Wang, wangzhonghua2691@sina.com) for data requests.

\section{Competing interests}

The authors have declared that no competing interest exists.

\section{Funding}

This study was supported by a grant from the Natural Science Foundation of Shanghai (19ZR1411200), a grant from Medicine Guidance Project of Shanghai Science and Technology Commission (KW1428) and a grant from China Anti-Cancer Association (HYXH202029). The funders had no role in the study design, collection and analysis of the data, decision to publish, or manuscript preparation.

\section{Authors' contributions}

Page 14/38 
ZHW, LC and XZZ designed the concept of the study. XZZ and XGL performed the experiments and collected data. $\mathrm{XZZ}$ and $\mathrm{BHH}$ were responsible for statistical analysis and interpretation. $\mathrm{XH}, \mathrm{YZJ}$ and ZMS contributed to the literature search and data collection and suggested additional experiments. XZZ wrote the final manuscript. LC, LY and ZHW revised the manuscript. All authors read and approved the final manuscript.

\section{Acknowledgments}

Not applicable.

\section{Author details}

${ }^{1}$ Department of Oncology, Shanghai Medical College, Fudan University, 130 Dong-An Road, Shanghai, 200032, P.R. China. ${ }^{2}$ Key Laboratory of Breast Cancer in Shanghai, Fudan University Shanghai Cancer Center, 270 Dong-An Road, Shanghai, 200032, P.R. China. ${ }^{3}$ Department of Breast Surgery, Fudan University Shanghai Cancer Center, 270 Dong-An Road, Shanghai, 200032, P.R. China. ${ }^{4}$ Department of Gastric Surgery, Fudan University Shanghai Cancer Center, 270 Dong-An Road, Shanghai, 200032, P.R. China. ${ }^{5}$ Precision Cancer Medicine Center, Shanghai, 200032, China. ${ }^{6}$ Institutes of Biomedical Science, Fudan University, Shanghai, 200032, China.

${ }^{7}$ Department of Breast Surgery, Fudan University Shanghai Cancer Center; Key Laboratory of Breast Cancer in Shanghai, Shanghai, 200032, China.

\section{References}

1. Harbeck N, Gnant M. Breast cancer. Lancet. 2017; 389: 1134-50.

2. Torre LA, Bray F, Siegel RL, Ferlay J, Lortet-Tieulent J, Jemal A. Global cancer statistics, 2012. CA Cancer J Clin. 2015; 65: 87-108.

3. Waks AG, Winer EP. Breast Cancer Treatment: A Review. JAMA. 2019; 321: 288-300.

4. Bianchini G, Balko JM, Mayer IA, Sanders ME, Gianni L. Triple-negative breast cancer: challenges and opportunities of a heterogeneous disease. Nat Rev Clin Oncol. 2016; 13: 674-90.

5. Jiang YZ, Ma D, Suo C, Shi J, Xue M, Hu X, et al. Genomic and Transcriptomic Landscape of TripleNegative Breast Cancers: Subtypes and Treatment Strategies. Cancer Cell. 2019; 35: 428-40.

6. Jiang YZ, Liu Y, Xiao Y, Hu X, Jiang L, Zuo WJ, et al. Molecular subtyping and genomic profiling expand precision medicine in refractory metastatic triple-negative breast cancer: the FUTURE trial. Cell Res. 2020.

7. Farmer H, McCabe N, Lord CJ, Tutt AN, Johnson DA, Richardson TB, et al. Targeting the DNA repair defect in BRCA mutant cells as a therapeutic strategy. Nature. 2005; 434: 917-21.

8. Francica P, Rottenberg S. Mechanisms of PARP inhibitor resistance in cancer and insights into the DNA damage response. Genome Med. 2018; 10: 101. 
9. Kim G, Ison G, McKee AE, Zhang H, Tang S, Gwise T, et al. FDA Approval Summary: Olaparib Monotherapy in Patients with Deleterious Germline BRCA-Mutated Advanced Ovarian Cancer Treated with Three or More Lines of Chemotherapy. Clin Cancer Res. 2015; 21: 4257-61.

10. Olaparib for Metastatic Breast Cancer in Patients with a Germline BRCA Mutation. N Engl J Med. 2017; 377: 1700.

11. Lord CJ, Ashworth A. PARP inhibitors: Synthetic lethality in the clinic. Science. 2017; 355: 1152-8.

12. Mateo J, Lord CJ, Serra V, Tutt A, Balmana J, Castroviejo-Bermejo M, et al. A decade of clinical development of PARP inhibitors in perspective. Ann Oncol. 2019; 30: 1437-47.

13. O'Leary B, Finn RS, Turner NC. Treating cancer with selective CDK4/6 inhibitors. Nat Rev Clin Oncol. 2016; 13: 417-30.

14. de Groot AF, Kuijpers CJ, Kroep JR. CDK4/6 inhibition in early and metastatic breast cancer: A review. Cancer Treat Rev. 2017; 60: 130-8.

15. Finn RS, Crown JP, Lang I, Boer K, Bondarenko IM, Kulyk SO, et al. The cyclin-dependent kinase 4/6 inhibitor palbociclib in combination with letrozole versus letrozole alone as first-line treatment of oestrogen receptor-positive, HER2-negative, advanced breast cancer (PALOMA-1/TRIO-18): a randomised phase 2 study. Lancet Oncol. 2015; 16: 25-35.

16. Raphael J, Helou J, Pritchard KI, Naimark DM. Palbociclib in hormone receptor positive advanced breast cancer: A cost-utility analysis. Eur J Cancer. 2017; 85: 146-54.

17. Kim ES. Abemaciclib: First Global Approval. Drugs. 2017; 77: 2063-70.

18. Asghar US, Barr AR, Cutts R, Beaney M, Babina I, Sampath D, et al. Single-Cell Dynamics Determines Response to CDK4/6 Inhibition in Triple-Negative Breast Cancer. Clin Cancer Res. 2017; 23: 5561-72.

19. Salvador-Barbero B, Alvarez-Fernandez M, Zapatero-Solana E, El BA, Menendez M, Lopez-Casas PP, et al. CDK4/6 Inhibitors Impair Recovery from Cytotoxic Chemotherapy in Pancreatic Adenocarcinoma. Cancer Cell. 2020; 37: 340-53.

20. Yi J, Liu C, Tao Z, Wang M, Jia Y, Sang X, et al. MYC status as a determinant of synergistic response to Olaparib and Palbociclib in ovarian cancer. Ebiomedicine. 2019; 43: 225-37.

21. Ashton JC. Drug combination studies and their synergy quantification using the Chou-Talalay method-letter. Cancer Res. 2015; 75: 2400.

22. Dimri GP, Lee X, Basile G, Acosta M, Scott G, Roskelley C, et al. A biomarker that identifies senescent human cells in culture and in aging skin in vivo. Proc Natl Acad Sci U S A. 1995; 92: 9363-7.

23. Sun R, Xie HY, Qian JX, Huang YN, Yang F, Zhang FL, et al. FBXO22 Possesses Both Protumorigenic and Antimetastatic Roles in Breast Cancer Progression. Cancer Res. 2018; 78: 5274-86.

24. Liu HY, Liu YY, Yang F, Zhang L, Zhang FL, Hu X, et al. Acetylation of MORC2 by NAT10 regulates cellcycle checkpoint control and resistance to DNA-damaging chemotherapy and radiotherapy in breast cancer. Nucleic Acids Res. 2020; 48: 3638-56.

25. Lu Q, Zhang FL, Lu DY, Shao ZM, Li DQ. USP9X stabilizes BRCA1 and confers resistance to DNAdamaging agents in human cancer cells. Cancer Med. 2019; 8: 6730-40. 
26. Pierce AJ, Johnson RD, Thompson LH, Jasin M. XRCC3 promotes homology-directed repair of DNA damage in mammalian cells. Genes Dev. 1999; 13: 2633-8.

27. Guglielmi C, Cerri I, Evangelista M, Collavoli A, Tancredi M, Aretini P, et al. Identification of two novel BRCA1-partner genes in the DNA double-strand break repair pathway. Breast Cancer Res Treat. 2013; 141: 515-22.

28. Zheng YZ, Xue MZ, Shen HJ, Li XG, Ma D, Gong Y, et al. PHF5A Epigenetically Inhibits Apoptosis to Promote Breast Cancer Progression. Cancer Res. 2018; 78: 3190-206.

29. Ran FA, Hsu PD, Wright J, Agarwala V, Scott DA, Zhang F. Genome engineering using the CRISPRCas9 system. Nat Protoc. 2013; 8: 2281-308.

30. Jin X, Ge LP, Li DQ, Shao ZM, Di GH, Xu XE, et al. LncRNA TROJAN promotes proliferation and resistance to $C D K 4 / 6$ inhibitor via CDK2 transcriptional activation in ER+ breast cancer. Mol Cancer. 2020; $19: 87$.

31. Zhu X, Chen L, Huang B, Wang Y, Ji L, Wu J, et al. The prognostic and predictive potential of Ki-67 in triple-negative breast cancer. Sci Rep. 2020; 10: 225.

32. Yang YL, Zhang Y, Li DD, Zhang FL, Liu HY, Liao XH, et al. RNF144A functions as a tumor suppressor in breast cancer through ubiquitin ligase activity-dependent regulation of stability and oncogenic functions of HSPA2. Cell Death Differ. 2020; 27: 1105-18.

33. Seluanov A, Mao Z, Gorbunova V. Analysis of DNA double-strand break (DSB) repair in mammalian cells. J Vis Exp. 2010.

34. Salvador-Barbero B, Alvarez-Fernandez M, Zapatero-Solana E, El BA, Menendez M, Lopez-Casas PP, et al. CDK4/6 Inhibitors Impair Recovery from Cytotoxic Chemotherapy in Pancreatic Adenocarcinoma. Cancer Cell. 2020; 37: 340-53.

35. McClendon AK, Dean JL, Rivadeneira DB, Yu JE, Reed CA, Gao E, et al. CDK4/6 inhibition antagonizes the cytotoxic response to anthracycline therapy. Cell Cycle. 2012; 11: 2747-55.

36. Scholz BA, Sumida N, de Lima C, Chachoua I, Martino M, Tzelepis I, et al. WNT signaling and AHCTF1 promote oncogenic MYC expression through super-enhancer-mediated gene gating. Nat Genet. 2019; 51: 1723-31.

37. Wang Q, Zhou Y, Rychahou P, Harris JW, Zaytseva YY, Liu J, et al. Deptor Is a Novel Target of Wnt/beta-Catenin/c-Myc and Contributes to Colorectal Cancer Cell Growth. Cancer Res. 2018; 78: 3163-75.

38. Wu X, Tu X, Joeng KS, Hilton MJ, Williams DA, Long F. Rac1 activation controls nuclear localization of beta-catenin during canonical Wnt signaling. Cell. 2008; 133: 340-53.

39. Taurin S, Sandbo N, Yau DM, Sethakorn N, Dulin NO. Phosphorylation of beta-catenin by PKA promotes ATP-induced proliferation of vascular smooth muscle cells. Am J Physiol Cell Physiol. 2008; 294: C1169-74.

40. Stamos JL, Weis WI. The $\beta$-catenin destruction complex. Cold Spring Harb Perspect Biol. 2013; 5: a7898. 
41. Noordermeer SM, van Attikum H. PARP Inhibitor Resistance: A Tug-of-War in BRCA-Mutated Cells. Trends Cell Biol. 2019; 29: 820-34.

42. Kohlhapp FJ, Kaufman HL. Molecular Pathways: Mechanism of Action for Talimogene Laherparepvec, a New Oncolytic Virus Immunotherapy. Clin Cancer Res. 2016; 22: 1048-54.

43. Ning J, Wakimoto H, Peters C, Martuza RL, Rabkin SD. Rad51 Degradation: Role in Oncolytic VirusPoly(ADP-Ribose) Polymerase Inhibitor Combination Therapy in Glioblastoma. J Natl Cancer Inst. 2017; 109: 1-13.

44. Barazas M, Gasparini A, Huang Y, Küçükosmanoğlu A, Annunziato S, Bouwman $P$, et al. Radiosensitivity Is an Acquired Vulnerability of PARPi-Resistant BRCA1-Deficient Tumors. Cancer Res. 2019; 79: 452-60.

45. Johnson SF, Cruz C, Greifenberg AK, Dust S, Stover DG, Chi D, et al. CDK12 Inhibition Reverses De Novo and Acquired PARP Inhibitor Resistance in BRCA Wild-Type and Mutated Models of TripleNegative Breast Cancer. Cell Rep. 2016; 17: 2367-81.

46. Tomimatsu N, Mukherjee B, Catherine HM, Ilcheva M, Vanessa CC, Louise HJ, et al. Phosphorylation of EXO1 by CDKs 1 and 2 regulates DNA end resection and repair pathway choice. Nat Commun. 2014; 5: 3561.

47. Ning JF, Stanciu M, Humphrey MR, Gorham J, Wakimoto H, Nishihara R, et al. Myc targeted CDK18 promotes ATR and homologous recombination to mediate PARP inhibitor resistance in glioblastoma. Nat Commun. 2019; 10: 2910.

48. Jiao S, Xia W, Yamaguchi H, Wei Y, Chen MK, Hsu JM, et al. PARP Inhibitor Upregulates PD-L1 Expression and Enhances Cancer-Associated Immunosuppression. Clin Cancer Res. 2017; 23: 371120.

49. Shen J, Zhao W, Ju Z, Wang L, Peng Y, Labrie M, et al. PARPi Triggers the STING-Dependent Immune Response and Enhances the Therapeutic Efficacy of Immune Checkpoint Blockade Independent of BRCAness. Cancer Res. 2019; 79: 311-9.

50. Liszczak G, Diehl KL, Dann GP, Muir TW. Acetylation blocks DNA damage-induced chromatin ADPribosylation. Nat Chem Biol. 2018; 14: 837-40.

51. Pulliam N, Fang F, Ozes AR, Tang J, Adewuyi A, Keer H, et al. An Effective Epigenetic-PARP Inhibitor Combination Therapy for Breast and Ovarian Cancers Independent of BRCA Mutations. Clin Cancer Res. 2018; 24: 3163-75.

52. Muvarak NE, Chowdhury K, Xia L, Robert C, Choi EY, Cai Y, et al. Enhancing the Cytotoxic Effects of PARP Inhibitors with DNA Demethylating Agents - A Potential Therapy for Cancer. Cancer Cell. 2016; 30: 637-50.

53. Wang H, Xiang D, Liu B, He A, Randle HJ, Zhang KX, et al. Inadequate DNA Damage Repair Promotes Mammary Transdifferentiation, Leading to BRCA1 Breast Cancer. Cell. 2019; 178: 135-51.

54. Karimaian A, Majidinia M, Bannazadeh BH, Yousefi B. The crosstalk between Wnt/ $\beta$-catenin signaling pathway with DNA damage response and oxidative stress: Implications in cancer therapy. DNA Repair (Amst). 2017; 51: 14-9. 
55. Prochownik EV, Li Y. The ever expanding role for c-Myc in promoting genomic instability. Cell Cycle. 2007; 6: 1024-9.

56. Meyer N, Penn LZ. Reflecting on 25 years with MYC. Nat Rev Cancer. 2008; 8: 976-90.

57. Wade M, Wahl GM. c-Myc, genome instability, and tumorigenesis: the devil is in the details. Curr Top Microbiol Immunol. 2006; 302: 169-203.

\section{Figures}



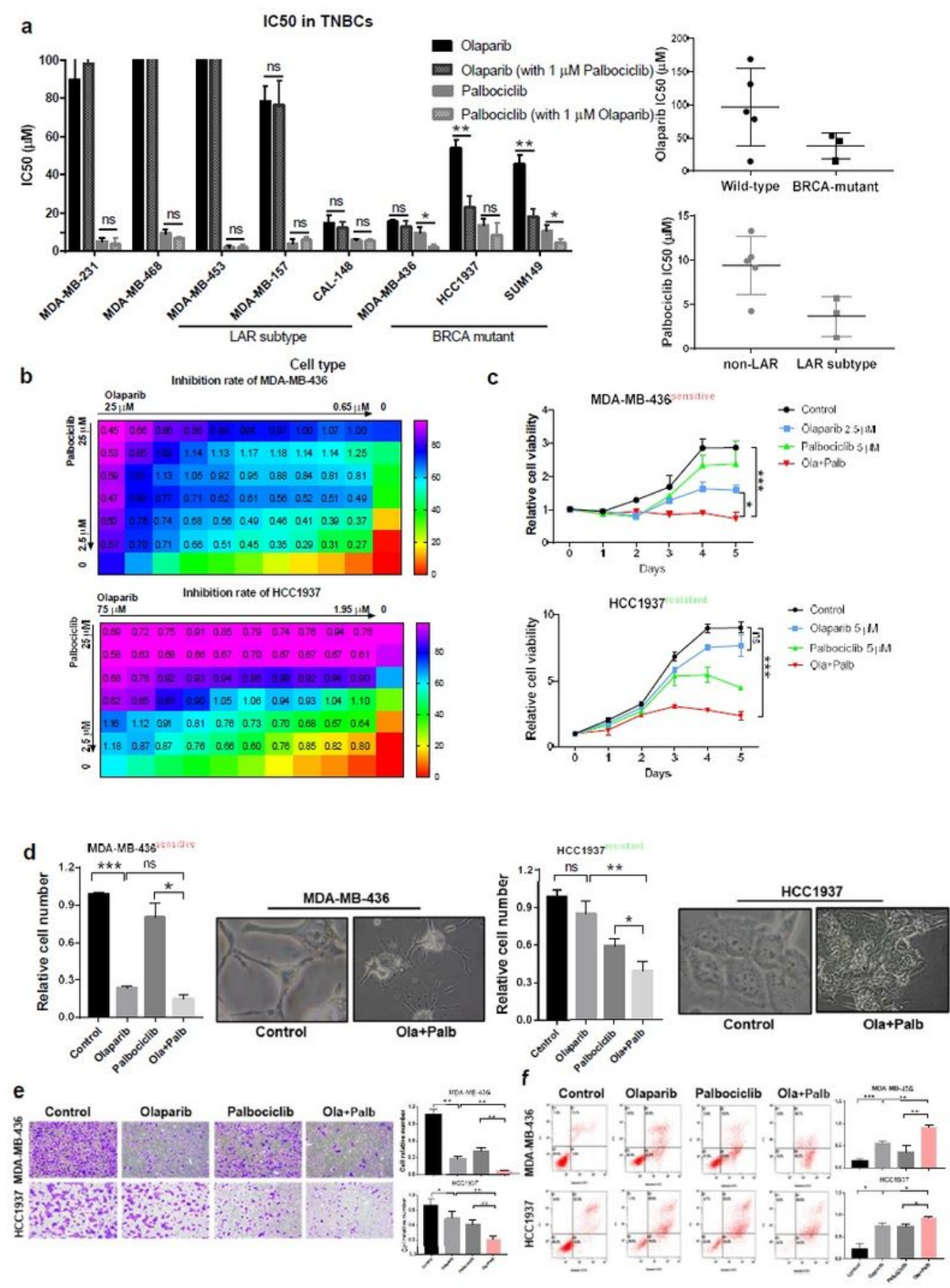

\section{Figure 1}

The synergistic effect of PARPi and CDK4/6i in BRCAmut/TNBCs. a The IC50 of olaparib and palbociclib in a panel of 8 TNBC cell lines. (Left) The four IC50s in each cell line are represented: olaparib, olaparib plus $1 \mu \mathrm{M}$ palbociclib, palbociclib and palbociclib plus $1 \mu \mathrm{M}$ olaparib. No data are displayed when IC50 was greater than $100 \mu \mathrm{M}$. (Right) The IC50 of olaparib was divided into two groups according to the BRCA mutation state, while that of palbociclib was based on the LAR subtype. b Inhibition rate and 
combination index (Cl) in (Up) MDA-MB-436 and (Down) HCC1937 cells at different drug concentrations $(\mu \mathrm{M})$. The color represents the degree of inhibition, and the displayed value represents the $\mathrm{Cl}$. Olaparib was diluted $2 / 3$ times from left to right, and the concentration of palbociclib $(\mu \mathrm{M})$ from top to bottom was: $25,20,15,10,5,2.5$, and 0 . c Cell proliferation curve of olaparib or palbociclib alone or in combination in (Up) the PARPi-sensitive cells MDA-MB-436 and (Down) PARPi-resistant cells HCC1937. $d$ Changes in relative cell numbers and cell morphology after three days of single-agent or combined treatment in MDA-MB-436 and HCC1937 cells. e Transwell migration assay in MDA-MB-436 and HCC1937 cells pretreated with drug as indicated for 3 days. The migration time for HCC1937 and MDAMB-436 cells was $18 \mathrm{~h}$ and $24 \mathrm{~h}$, respectively. $\mathrm{f}$ Apoptosis evaluation after three days of single-agent or combined treatment in MDA-MB-436 and HCC1937 cells. c(olaparib) $=15 \mu \mathrm{M}, \mathrm{c}$ (palbociclib) $=15 \mu \mathrm{M}$. Student's t-test; ${ }^{\star \star *} p<0.001,{ }^{* \star} p<0.01,{ }^{*} p<0.05$; ns, not significant. The data are presented as the means \pm SEMs. Ola: Olaparib; Palb: Palbociclib. 


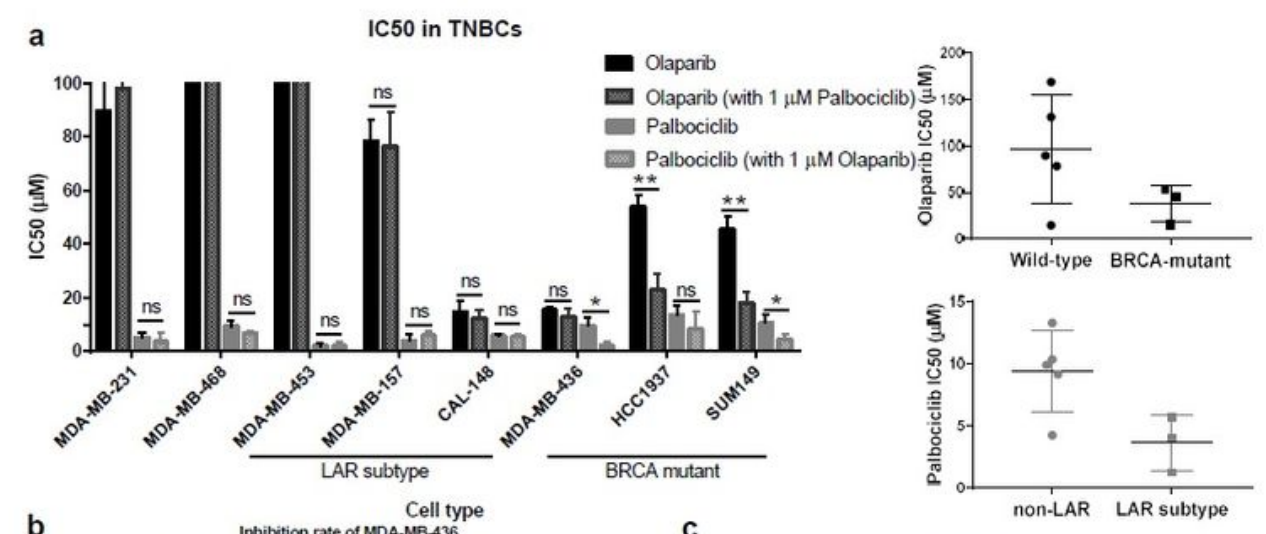

b

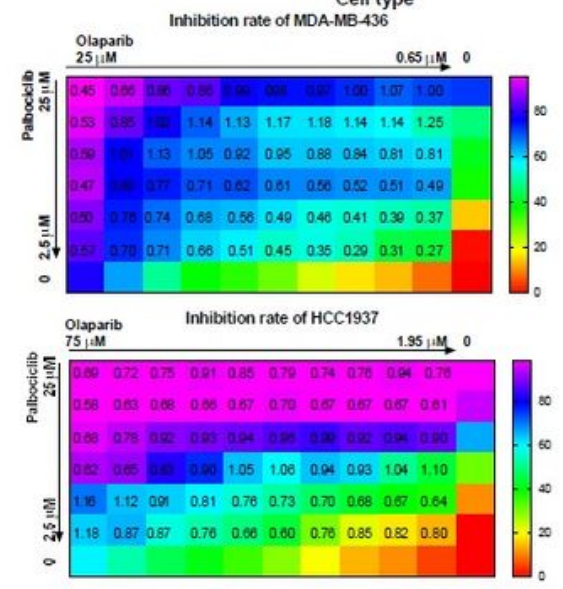

c
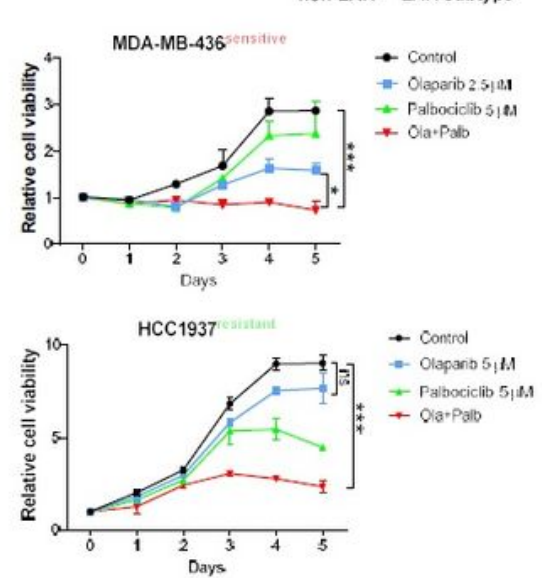
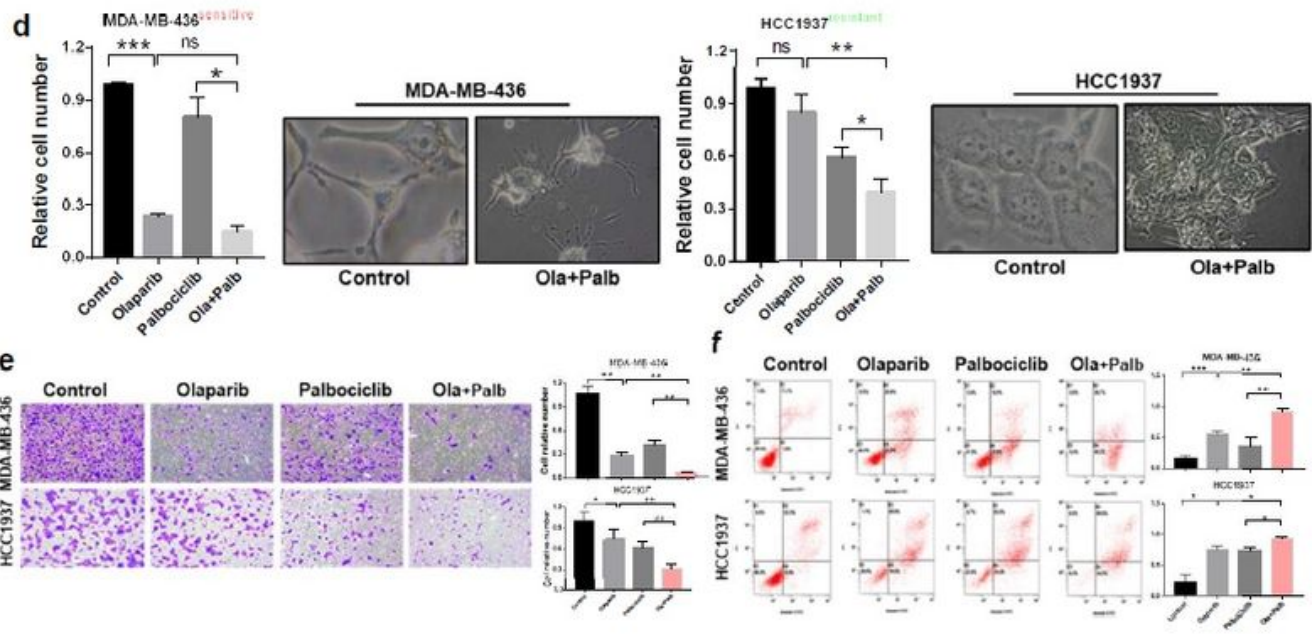

\section{Figure 1}

The synergistic effect of PARPi and CDK4/6i in BRCAmut/TNBCs. a The IC50 of olaparib and palbociclib in a panel of 8 TNBC cell lines. (Left) The four IC50s in each cell line are represented: olaparib, olaparib plus $1 \mu \mathrm{M}$ palbociclib, palbociclib and palbociclib plus $1 \mu \mathrm{M}$ olaparib. No data are displayed when IC50 was greater than $100 \mu \mathrm{M}$. (Right) The IC50 of olaparib was divided into two groups according to the BRCA mutation state, while that of palbociclib was based on the LAR subtype. b Inhibition rate and 
combination index (Cl) in (Up) MDA-MB-436 and (Down) HCC1937 cells at different drug concentrations $(\mu \mathrm{M})$. The color represents the degree of inhibition, and the displayed value represents the $\mathrm{Cl}$. Olaparib was diluted $2 / 3$ times from left to right, and the concentration of palbociclib $(\mu \mathrm{M})$ from top to bottom was: $25,20,15,10,5,2.5$, and 0 . c Cell proliferation curve of olaparib or palbociclib alone or in combination in (Up) the PARPi-sensitive cells MDA-MB-436 and (Down) PARPi-resistant cells HCC1937. $d$ Changes in relative cell numbers and cell morphology after three days of single-agent or combined treatment in MDA-MB-436 and HCC1937 cells. e Transwell migration assay in MDA-MB-436 and HCC1937 cells pretreated with drug as indicated for 3 days. The migration time for HCC1937 and MDAMB-436 cells was $18 \mathrm{~h}$ and $24 \mathrm{~h}$, respectively. $\mathrm{f}$ Apoptosis evaluation after three days of single-agent or combined treatment in MDA-MB-436 and HCC1937 cells. c(olaparib) $=15 \mu \mathrm{M}, \mathrm{c}$ (palbociclib) $=15 \mu \mathrm{M}$. Student's t-test; ${ }^{\star \star *} p<0.001,{ }^{* \star} p<0.01,{ }^{*} p<0.05$; ns, not significant. The data are presented as the means \pm SEMs. Ola: Olaparib; Palb: Palbociclib. 

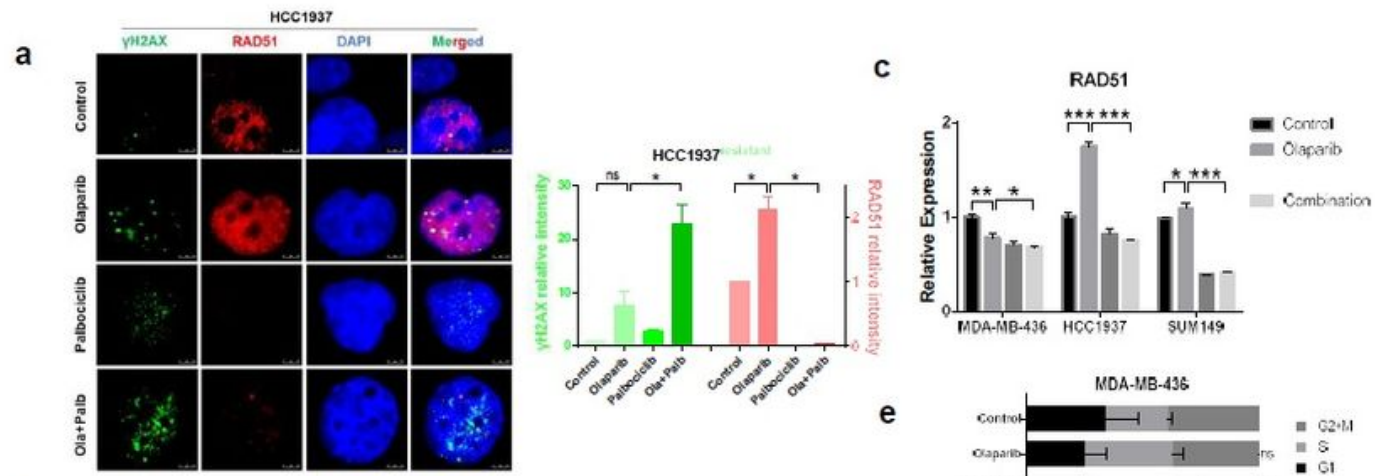

b
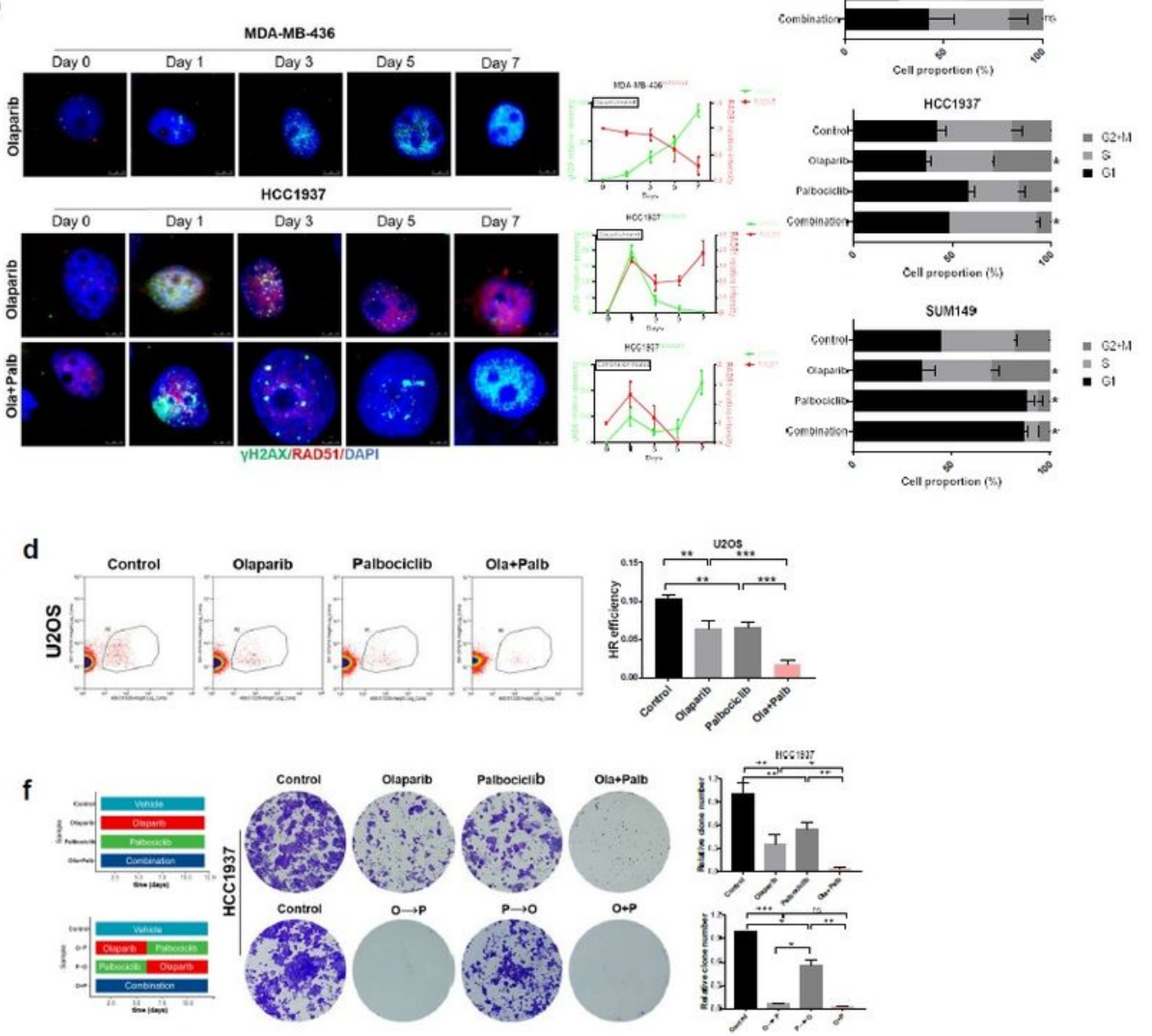

\section{Figure 2}

The combination of PARPi and CDK4/6i results in irreversible lethal DNA damage. a (Left) Representative images of immunofluorescent staining of DAPI, YH2AX and RAD51 in HCC1937 cells treated with vehicle, $5 \mu \mathrm{M}$ olaparib, $5 \mu \mathrm{M}$ palbociclib or their combination for $72 \mathrm{~h}$. (Right) The quantification of $\mathrm{YH} 2 \mathrm{AX}$ and RAD51 signal intensity was evaluated by ImageJ. Scale bar, $7.5 \mu \mathrm{m}$. b (Left) Representative images at different treatment time points of BRCAmut/TNBC cells stained with DAPI, $y H 2 A X$ and RAD51. (Up) MDA- 
MD-436 cells treated with olaparib alone. (Down) HCC1937 cells treated with olaparib alone or olaparib plus palbociclib. (Right) The signal intensity of $\mathrm{YH} 2 \mathrm{AX}$ and RAD51 in cells changing over time. Scale bar, $7.5 \mu \mathrm{m}$. c Quantitative reverse transcription PCR analysis of RAD51 mRNA expression in BRCAmut/TNBC cells treated with drugs as indicated in (A) for $72 \mathrm{~h}$. $d$ Efficiency of homologous recombination (HR) in U2OS cells treated with vehicle, $20 \mu \mathrm{M}$ olaparib, $5 \mu \mathrm{M}$ palbociclib or their combination for $72 \mathrm{~h} ; \mathrm{n}=3$ independent assays. e The percentage of cells in different phases of the cell cycle was analyzed by flow cytometry. The treatment of cells was as referred in (A). The p-value here is the t-test of the G1 phase of each group relative to the control group. $f$ Colony formation assays of HCC1937 cell lines. The effect of the 6-day Olaparib followed by 6-day palbociclib sequential applicationखO-P囚and its reverse (P-O) effect were tested in HCC1937 cells. (Left) Pattern diagram of the sequential administration. (Middle) Representative images of colony formation assays. (Right) Quantification of colony formation. Student's t-test; ${ }^{* \star \star} p<0.001,{ }^{* \star} p<0.01,{ }^{\star} p<0.05$; ns, not significant. The data are presented as the means \pm SEMs. Con: Control; Ola: Olaparib; Palb: Palbociclib. 

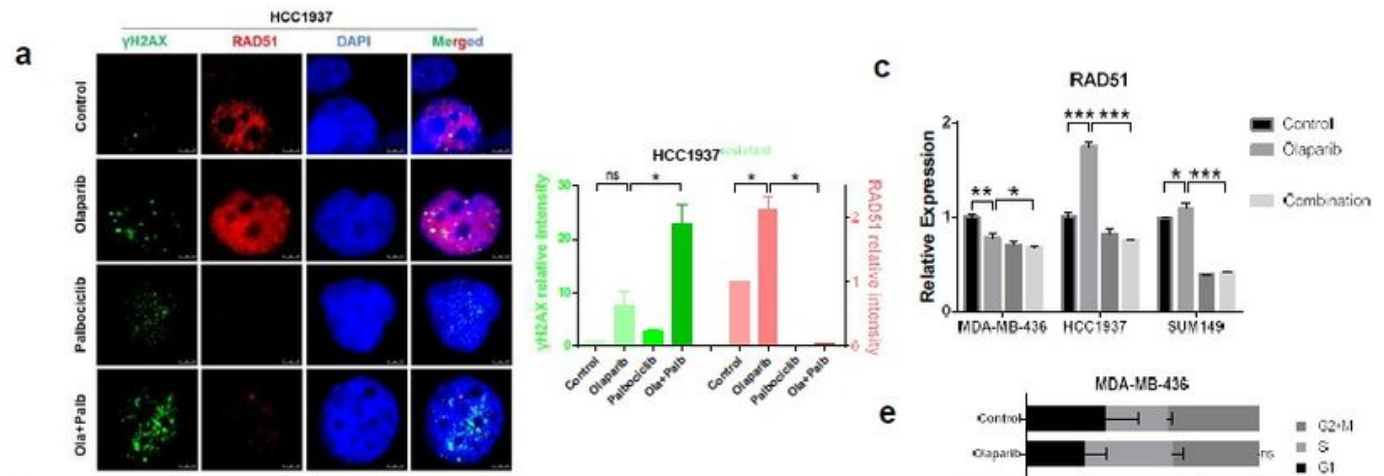

b
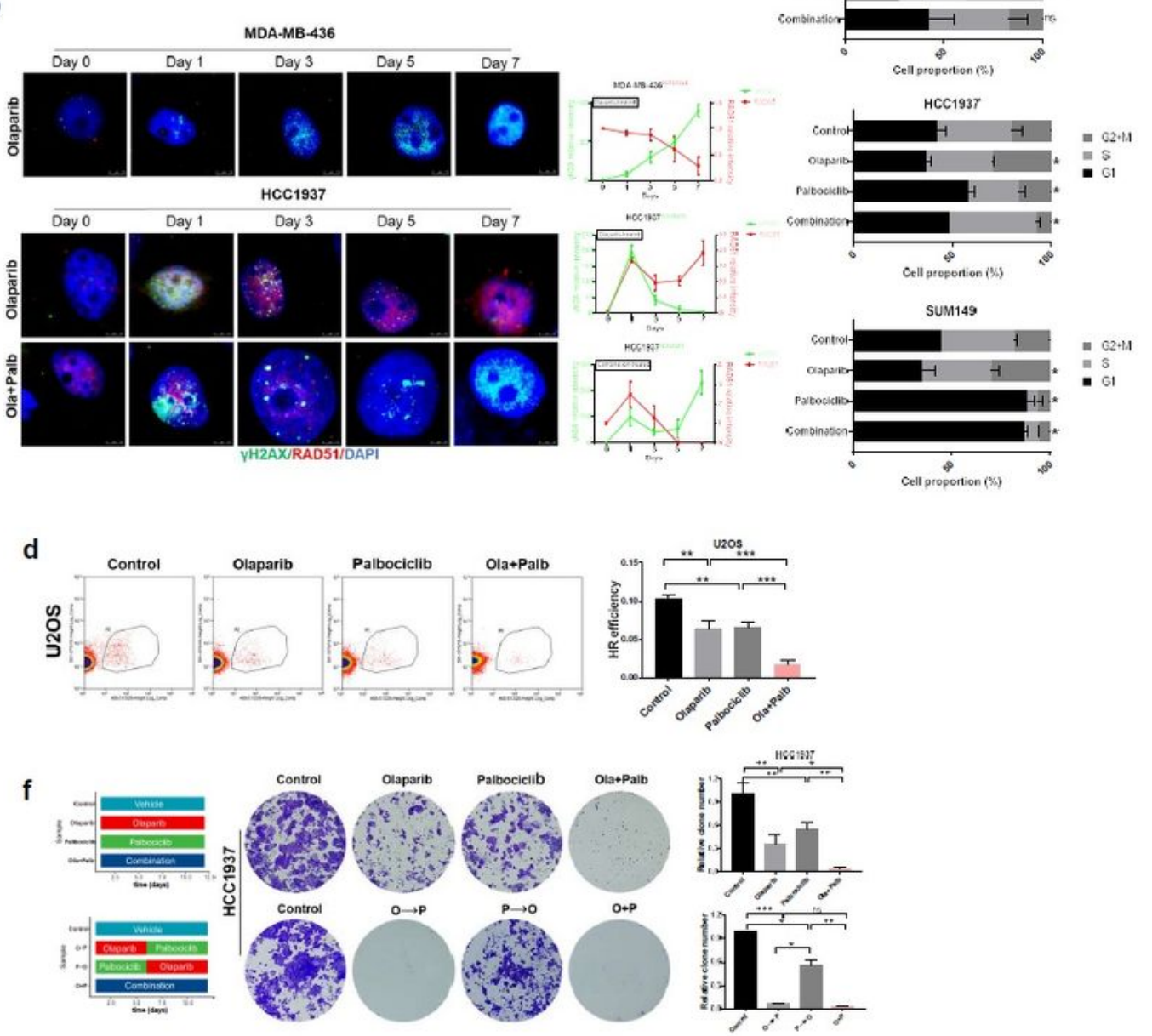

\section{Figure 2}

The combination of PARPi and CDK4/6i results in irreversible lethal DNA damage. a (Left) Representative images of immunofluorescent staining of DAPI, YH2AX and RAD51 in HCC1937 cells treated with vehicle, $5 \mu \mathrm{M}$ olaparib, $5 \mu \mathrm{M}$ palbociclib or their combination for $72 \mathrm{~h}$. (Right) The quantification of $\mathrm{YH} 2 \mathrm{AX}$ and RAD51 signal intensity was evaluated by ImageJ. Scale bar, $7.5 \mu \mathrm{m}$. b (Left) Representative images at different treatment time points of BRCAmut/TNBC cells stained with DAPI, $y H 2 A X$ and RAD51. (Up) MDA- 
MD-436 cells treated with olaparib alone. (Down) HCC1937 cells treated with olaparib alone or olaparib plus palbociclib. (Right) The signal intensity of $\mathrm{YH} 2 \mathrm{AX}$ and RAD51 in cells changing over time. Scale bar, $7.5 \mu \mathrm{m}$. c Quantitative reverse transcription PCR analysis of RAD51 mRNA expression in BRCAmut/TNBC cells treated with drugs as indicated in (A) for $72 \mathrm{~h}$. $d$ Efficiency of homologous recombination (HR) in U2OS cells treated with vehicle, $20 \mu \mathrm{M}$ olaparib, $5 \mu \mathrm{M}$ palbociclib or their combination for $72 \mathrm{~h} ; \mathrm{n}=3$ independent assays. e The percentage of cells in different phases of the cell cycle was analyzed by flow cytometry. The treatment of cells was as referred in (A). The p-value here is the t-test of the G1 phase of each group relative to the control group. $f$ Colony formation assays of HCC1937 cell lines. The effect of the 6-day Olaparib followed by 6-day palbociclib sequential applicationखO-P囚and its reverse (P-O) effect were tested in HCC1937 cells. (Left) Pattern diagram of the sequential administration. (Middle) Representative images of colony formation assays. (Right) Quantification of colony formation. Student's t-test; ${ }^{* \star \star} p<0.001,{ }^{* \star} p<0.01,{ }^{\star} p<0.05$; ns, not significant. The data are presented as the means \pm SEMs. Con: Control; Ola: Olaparib; Palb: Palbociclib. 
a

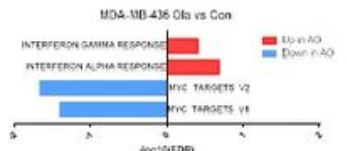

b

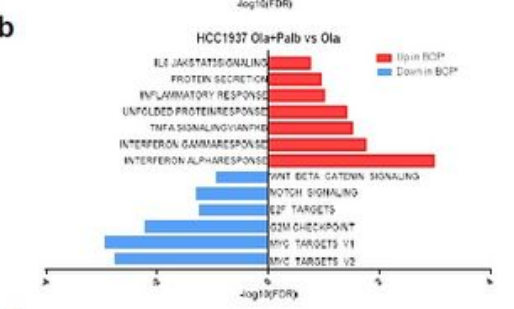

d

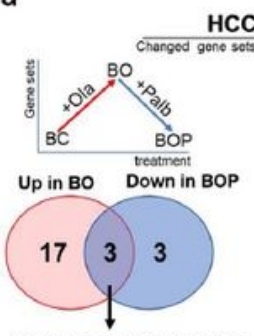

WNT BETA CATENIN SIGNALING G2M CHECKPONT
CC1937

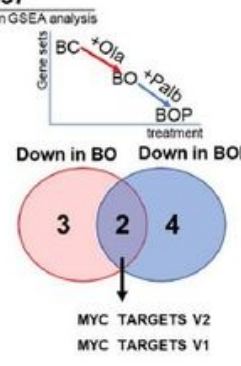

C
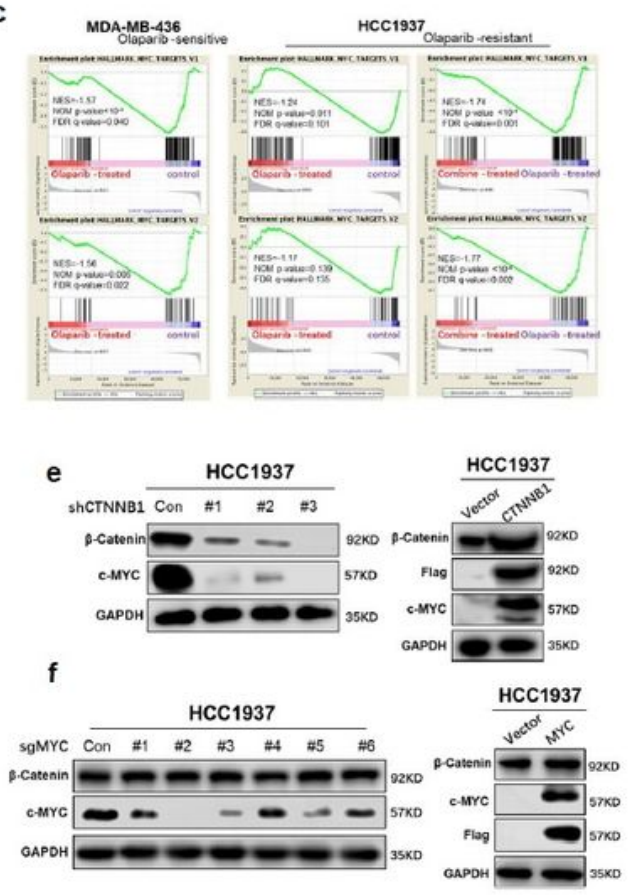

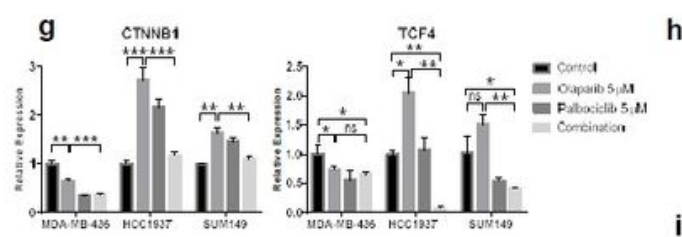

h
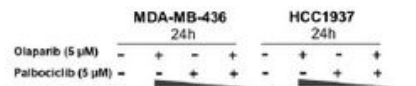

rearnin $=$

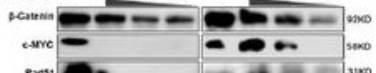

R20051 b-

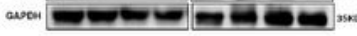

Axinz

Garc

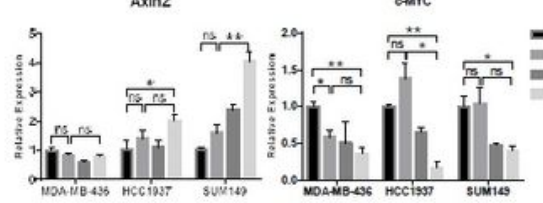

HCC1937 $12 \mathrm{~h}$

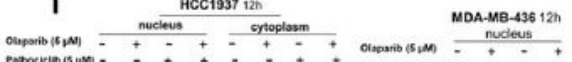

j

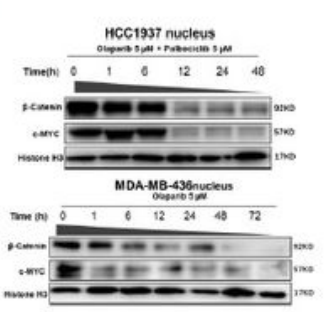

k

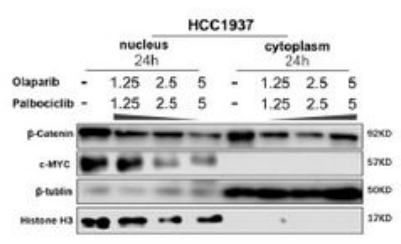

1

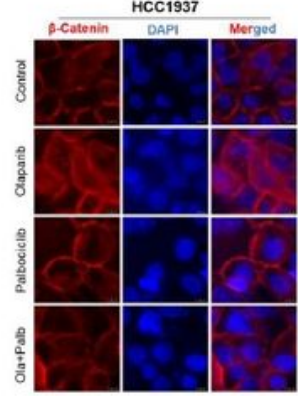

\section{Figure 3}

The combined use of PARPi and CDK4/6i inhibits MYC expression through the Wnt pathway. a GSEA results in olaparib-treated PARPi-sensitive MDA-MB-436 cells (AO) compared to vehicle-treated cells (AC). b GSEA results in combination-treated PARPi-resistant HCC1937 cells (BOP) compared to oaparib-treated cells (BO). c GSEA of MYC gene signatures in MDA-MB-436 (left panels) and HCC1937 (right panels) cells with different treatments. $d$ (Left) The overlap of Hallmark gene sets by GSEA which were downregulated 
in combination-treated cells (BOP) but upregulated in olaparib-treated resistant HCC1937 cells (BO). (Right) The overlap of Hallmark gene sets by GSEA that were both downregulated in BO and BOP. e Western blot (WB) analysis showed levels of c-myc in CTNNB1-knockdown (CTNNB1 KD) or overexpression (CTNNB1 OE) HCC1937 cells. f WB analysis showed levels of $\beta$-catenin in MYC-knockout (MYC KO) or overexpression (MYC OE) HCC1937 cells. g Quantitative reverse transcription PCR analysis of CTNNB1, TCF4, Axin2 and MYC in MDA-MB-436, HCC1937 and SUM149 BRCAmut/TNBC cell lines treated with drugs as indicated for $24 \mathrm{~h}$. Mean \pm S.D. for three independent experiments. $\mathrm{h}$ WB analysis showed the total levels of $\beta$-catenin, c-myc and Rad51 in MDA-MB-436 and HCC1937 cells treated with drugs as indicated for $24 \mathrm{~h}$. i WB analysis showed the nucleoplasmic distribution of $\beta$-catenin and the change in the Wnt pathway upon treatment with drugs as indicated for $12 \mathrm{~h}$. j Changes in nuclear $\beta$ catenin and c-myc protein levels over time under the indicated treatment. $\mathrm{k}$ Changes in the nucleoplasmic distribution of $\beta$-catenin and c-myc under different drug concentrations for $24 \mathrm{~h}$. I Immunofluorescence analysis of the nucleoplasmic distribution of $\beta$-catenin in HCC1937 cells treated with drugs as indicated for $12 \mathrm{~h}$. Scale bar, $7.5 \mu \mathrm{m}$. Student's t-test; ${ }^{\star \star \star} \mathrm{p}<0.001$, ${ }^{\star \star} \mathrm{p}<0.01,{ }^{*} \mathrm{p}<0.05$; ns, not significant. The data are presented as the means \pm SEMs. Con: Control; Ola: Olaparib; Palb: Palbociclib. 
a

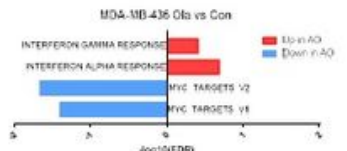

b

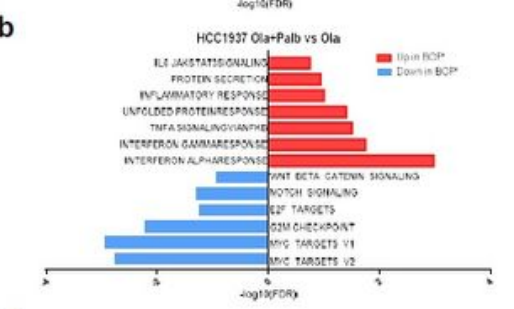

d

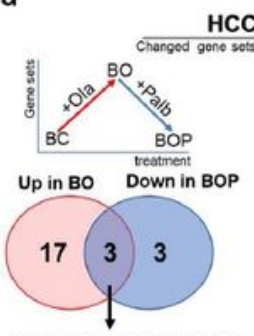

WNT BETA CATENIN SIGNALING G2M CHECKPONT
CC1937

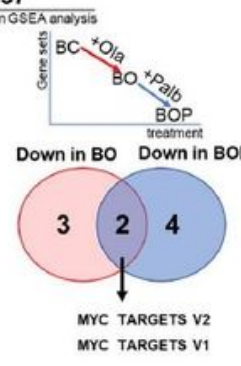

C
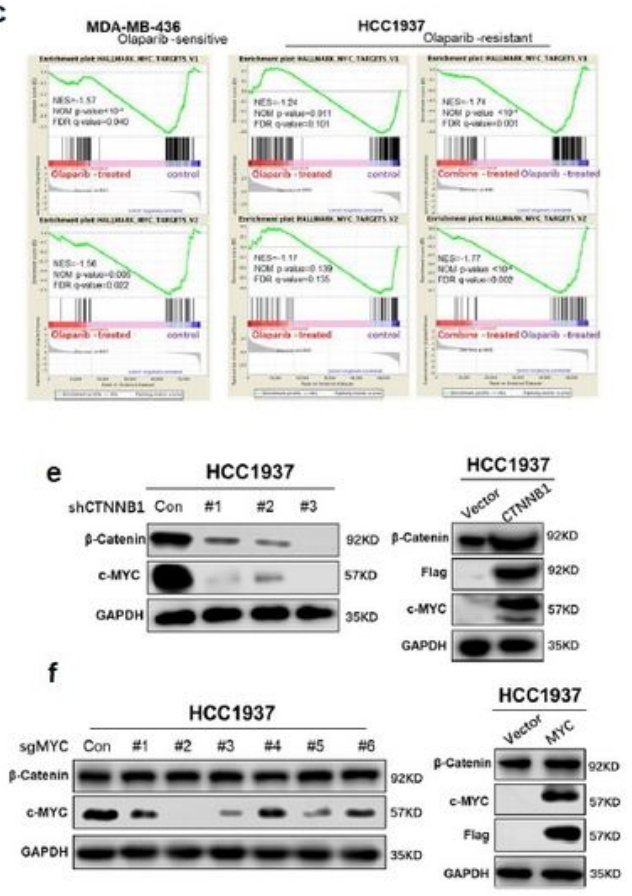

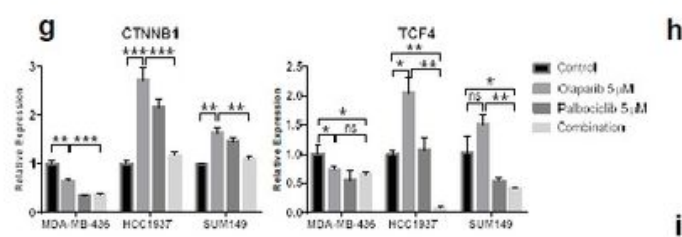

h
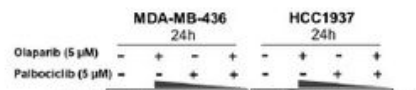

rearnin $=$

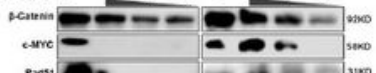

R200s (b-

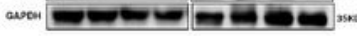

Axinz

Garc

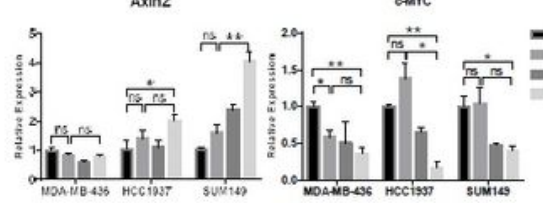

HCC1937 $12 \mathrm{~h}$

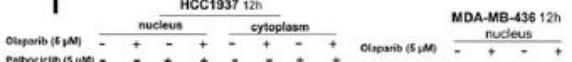

j

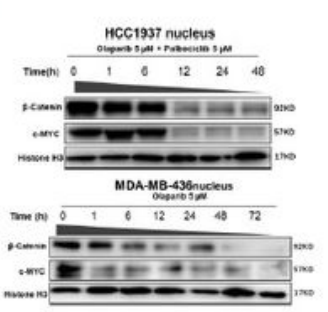

k

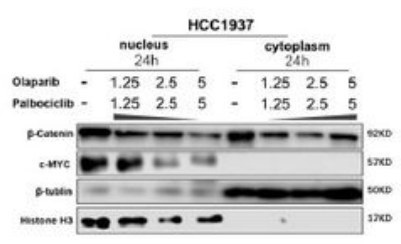

1

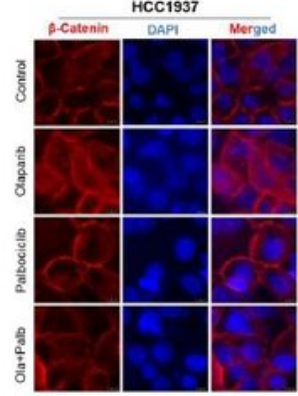

\section{Figure 3}

The combined use of PARPi and CDK4/6i inhibits MYC expression through the Wnt pathway. a GSEA results in olaparib-treated PARPi-sensitive MDA-MB-436 cells (AO) compared to vehicle-treated cells (AC). b GSEA results in combination-treated PARPi-resistant HCC1937 cells (BOP) compared to oaparib-treated cells (BO). c GSEA of MYC gene signatures in MDA-MB-436 (left panels) and HCC1937 (right panels) cells with different treatments. $d$ (Left) The overlap of Hallmark gene sets by GSEA which were downregulated 
in combination-treated cells (BOP) but upregulated in olaparib-treated resistant HCC1937 cells (BO). (Right) The overlap of Hallmark gene sets by GSEA that were both downregulated in BO and BOP. e Western blot (WB) analysis showed levels of c-myc in CTNNB1-knockdown (CTNNB1 KD) or overexpression (CTNNB1 OE) HCC1937 cells. f WB analysis showed levels of $\beta$-catenin in MYC-knockout (MYC KO) or overexpression (MYC OE) HCC1937 cells. g Quantitative reverse transcription PCR analysis of CTNNB1, TCF4, Axin2 and MYC in MDA-MB-436, HCC1937 and SUM149 BRCAmut/TNBC cell lines treated with drugs as indicated for $24 \mathrm{~h}$. Mean \pm S.D. for three independent experiments. $\mathrm{h}$ WB analysis showed the total levels of $\beta$-catenin, c-myc and Rad51 in MDA-MB-436 and HCC1937 cells treated with drugs as indicated for $24 \mathrm{~h}$. i WB analysis showed the nucleoplasmic distribution of $\beta$-catenin and the change in the Wnt pathway upon treatment with drugs as indicated for $12 \mathrm{~h}$. j Changes in nuclear $\beta$ catenin and c-myc protein levels over time under the indicated treatment. $\mathrm{k}$ Changes in the nucleoplasmic distribution of $\beta$-catenin and c-myc under different drug concentrations for $24 \mathrm{~h}$. I Immunofluorescence analysis of the nucleoplasmic distribution of $\beta$-catenin in HCC1937 cells treated with drugs as indicated for $12 \mathrm{~h}$. Scale bar, $7.5 \mu \mathrm{m}$. Student's t-test; ${ }^{\star \star \star} \mathrm{p}<0.001$, ${ }^{\star \star} \mathrm{p}<0.01,{ }^{*} \mathrm{p}<0.05$; ns, not significant. The data are presented as the means \pm SEMs. Con: Control; Ola: Olaparib; Palb: Palbociclib. 

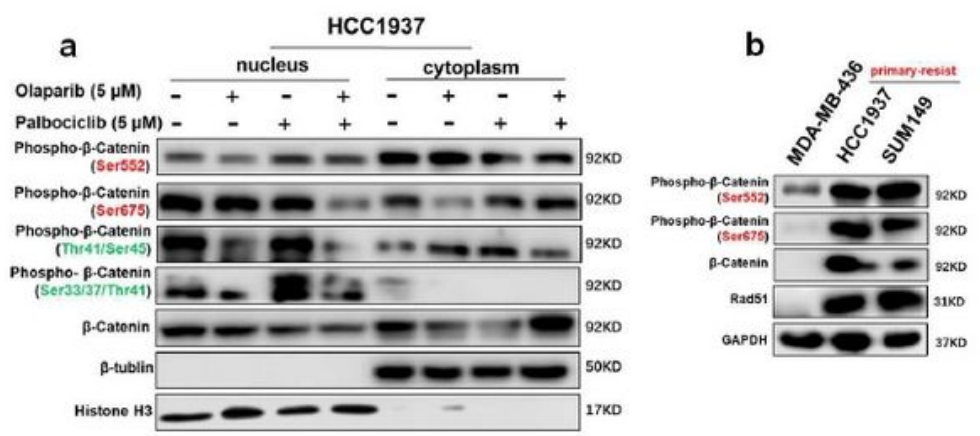

C
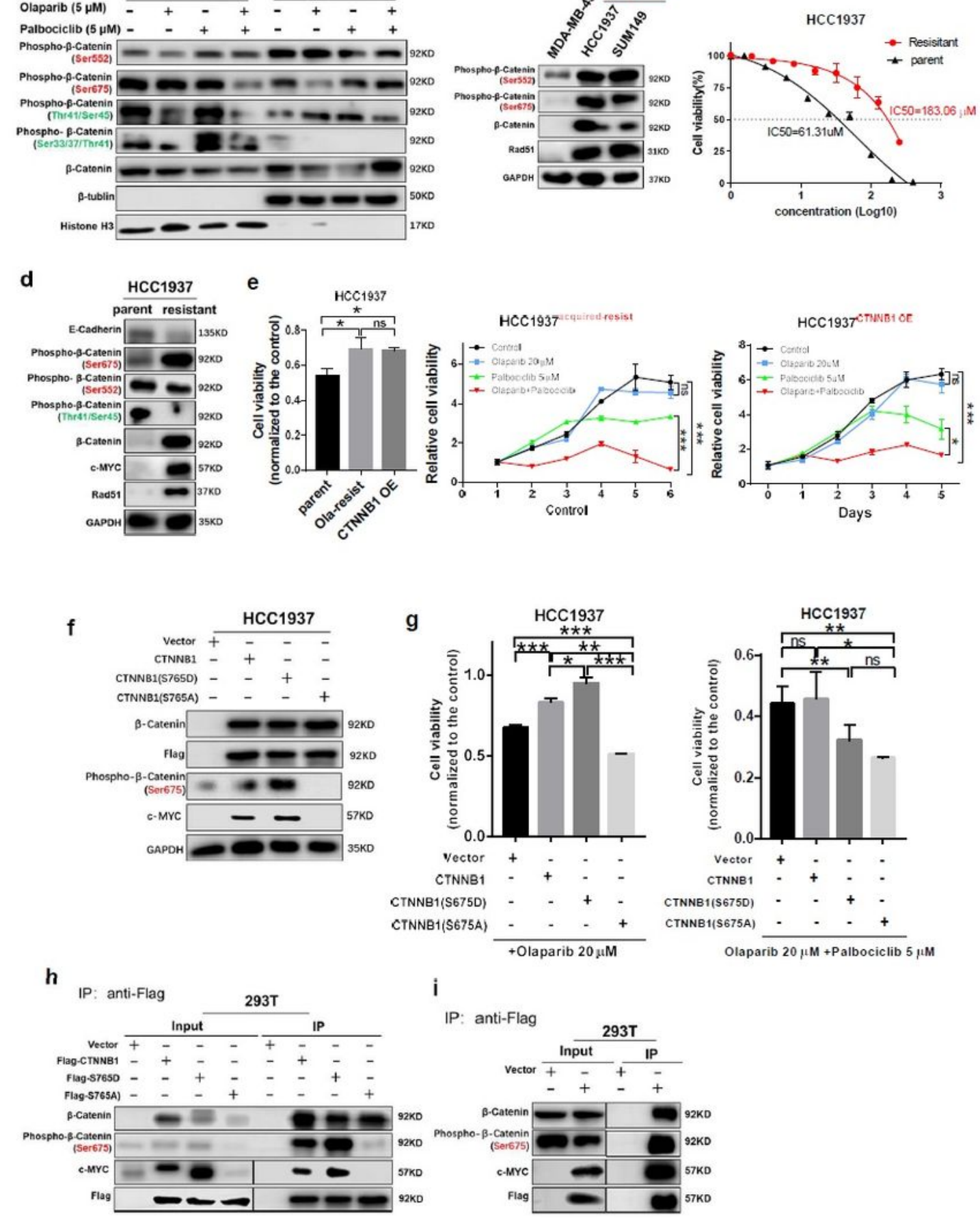

i

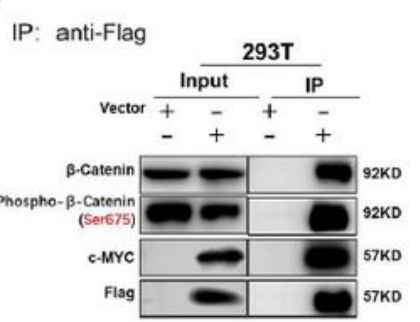

\section{Figure 4}

Ser675 phosphorylation of $\beta$-catenin in the Wnt pathway mediates resistance to PARPi but can be inhibited by CDK4/6i. a The phosphorylation level of specific sites of $\beta$-catenin in cells treated with drugs as indicated for $12 \mathrm{~h}$. The phosphorylation sites marked in green negatively regulates the Wnt pathway while those marked in red promote its nuclear translocation, thereby positively regulating the Wnt pathway. b Western blot (WB) analysis showing the levels of $\beta$-catenin, phosphorylated $\beta$-catenin ( $p \beta$ - 
cateninSer552 and p $\beta$-cateninSer675) and RAD51 (a homologous recombination repair marker) in MDAMB-436 and HCC1937 and SUM149 cells. c CCK-8 analysis of parental HCC1937 cells and the cells with acquired resistance to olaparib under different concentrations of olaparib. $d$ Expression difference in Wnt signaling pathway related proteins and Rad51 between parental HCC1937 and acquired olaparibresistant cells assessed by WB analysis. e (Left)The viability of parental HCC1937 cells, acquired olaparib-resistant HCC1937 cells and CTNNB1 OE HCC1937 cells under treatment as indicated. (Right) Growth curves of HCC1937 and CTNNB1 OE HCC1937 cells with acquired olaparib resistance treated with drugs as indicated. $f$ WB analysis of $\beta$-catenin, $p \beta$-cateninSer675 and c-myc protein levels in CTNNB1 KD HCC1937 cells with or without $\beta$-catenin mutant overexpression. $g$ (Left) The viability of the control and HCC1937 cells expressing $\beta$-cateninWT, mutant $\beta$-cateninS675D or $\beta$-cateninS675A under treatment as indicated. (Right) The four cell types under combined treatment for 5 days. $\mathrm{h}$ and i Co-IP of c-myc with $\beta$ catenin and its mutants in HEK293T cells. Student's t-test; ${ }^{* \star \star} p<0.001,{ }^{\star \star} p<0.01,{ }^{\star} p<0.05$; ns, not significant. The data are presented as the means \pm SEMs. Con: Control; Ola: Olaparib; Palb: Palbociclib. 

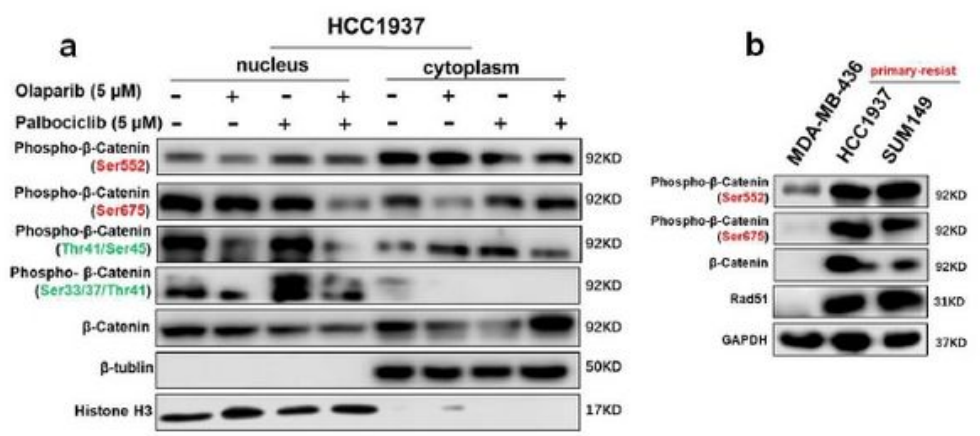

C
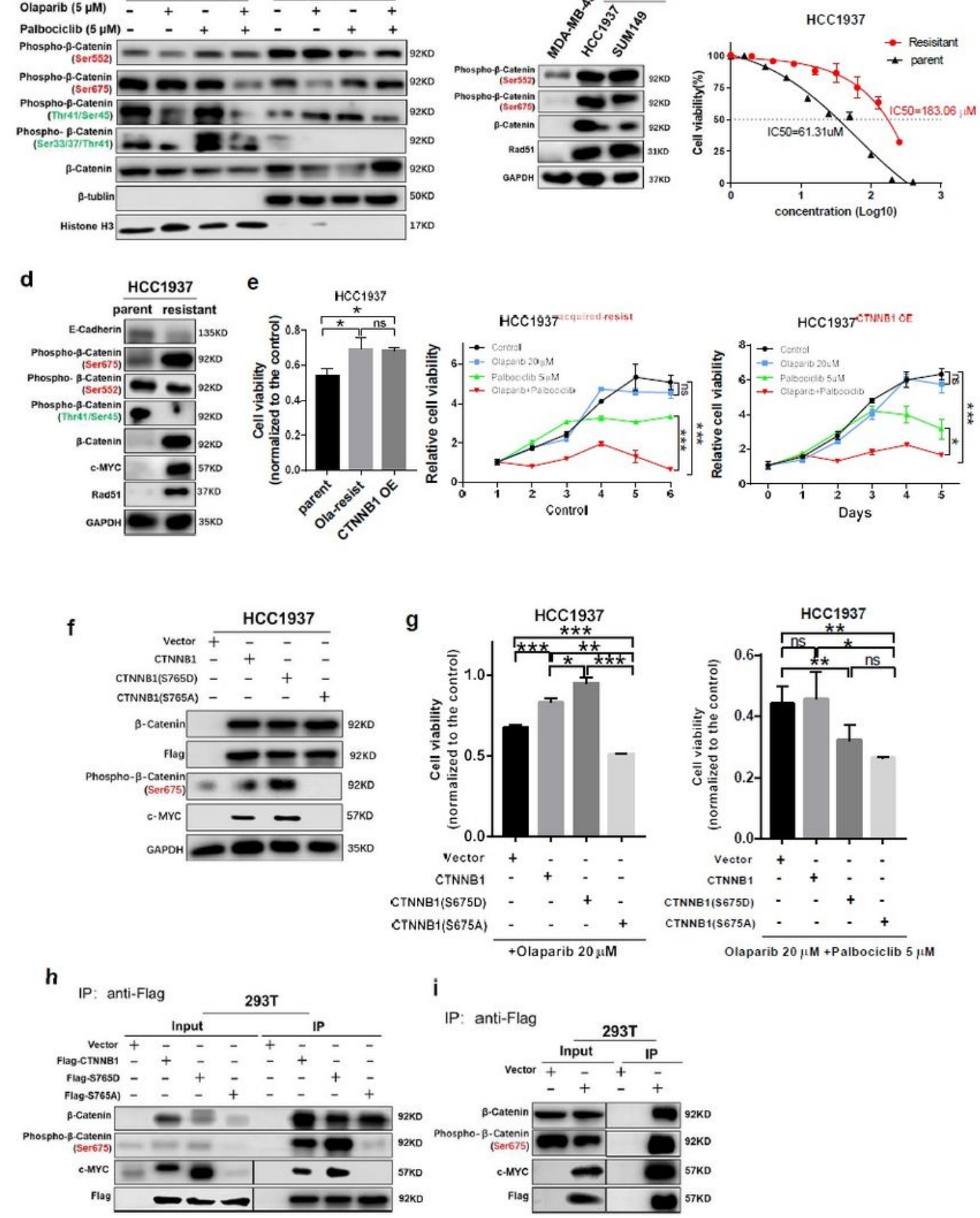

i

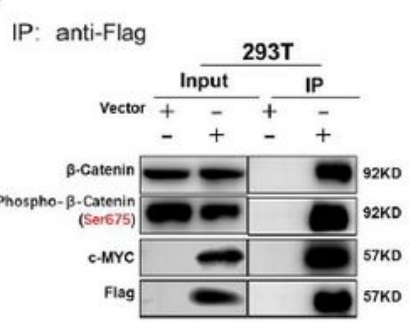

\section{Figure 4}

Ser675 phosphorylation of $\beta$-catenin in the Wnt pathway mediates resistance to PARPi but can be inhibited by CDK4/6i. a The phosphorylation level of specific sites of $\beta$-catenin in cells treated with drugs as indicated for $12 \mathrm{~h}$. The phosphorylation sites marked in green negatively regulates the Wnt pathway while those marked in red promote its nuclear translocation, thereby positively regulating the Wnt pathway. b Western blot (WB) analysis showing the levels of $\beta$-catenin, phosphorylated $\beta$-catenin ( $p \beta$ - 
cateninSer552 and p $\beta$-cateninSer675) and RAD51 (a homologous recombination repair marker) in MDAMB-436 and HCC1937 and SUM149 cells. c CCK-8 analysis of parental HCC1937 cells and the cells with acquired resistance to olaparib under different concentrations of olaparib. $d$ Expression difference in Wnt signaling pathway related proteins and Rad51 between parental HCC1937 and acquired olaparibresistant cells assessed by WB analysis. e (Left)The viability of parental HCC1937 cells, acquired olaparib-resistant HCC1937 cells and CTNNB1 OE HCC1937 cells under treatment as indicated. (Right) Growth curves of HCC1937 and CTNNB1 OE HCC1937 cells with acquired olaparib resistance treated with drugs as indicated. $f$ WB analysis of $\beta$-catenin, $p \beta$-cateninSer675 and c-myc protein levels in CTNNB1 KD HCC1937 cells with or without $\beta$-catenin mutant overexpression. $g$ (Left) The viability of the control and HCC1937 cells expressing $\beta$-cateninWT, mutant $\beta$-cateninS675D or $\beta$-cateninS675A under treatment as indicated. (Right) The four cell types under combined treatment for 5 days. $\mathrm{h}$ and i Co-IP of c-myc with $\beta$ catenin and its mutants in HEK293T cells. Student's t-test; ${ }^{\star \star \star} p<0.001,{ }^{\star \star} p<0.01$, ${ }^{\star} p<0.05$; ns, not significant. The data are presented as the means \pm SEMs. Con: Control; Ola: Olaparib; Palb: Palbociclib. 

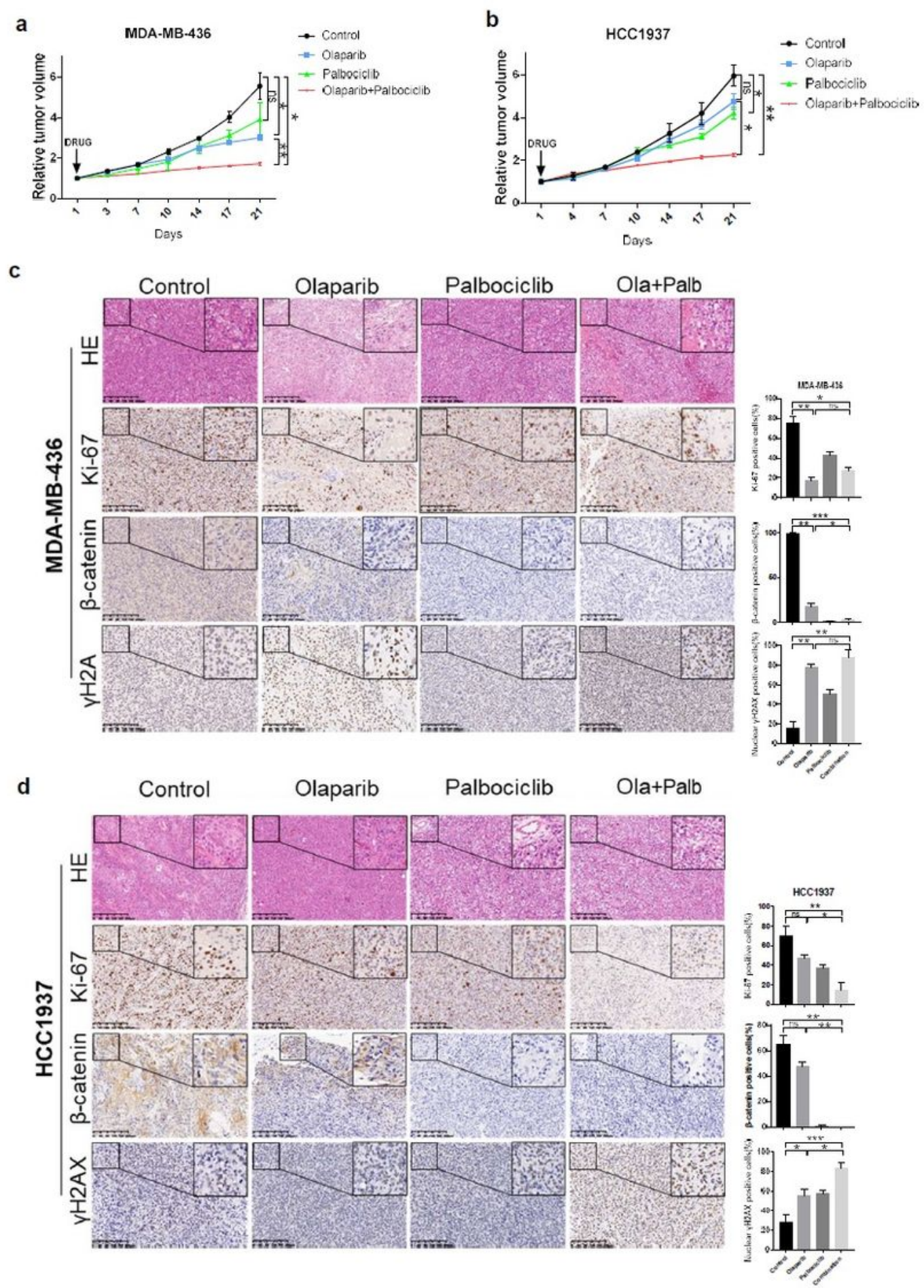

\section{Figure 5}

The combined treatment of Olaparib and Palbociclib is effective in vivo. $a$ and $b$ Tumor growth curves of (A) MDA-MB-436 and (B) HCC1937 xenografted NOD-SCID mice treated with olaparib (50 $\mathrm{mg} / \mathrm{kg} /$ day) and palbociclib (100 mg/kg/day), either alone or in combination, for 21 days. The arrow indicates the start of treatment. $c$ and d Representative images of immunohistochemical staining as indicated in the xenografted tumors ( $n \geq 3$ per treatment group) treated with olaparib and palbociclib either as single 
agents or in combination for 21 days. Scale bar, $200 \mu \mathrm{m}$. The small image inside is 4 times larger than the big image. Student's t-test; ${ }^{\star \star \star} p<0.001,{ }^{\star \star} p<0.01,{ }^{\star} p<0.05$; ns, not significant. The data are presented as the means \pm SEMs. Con: Control; Ola: Olaparib; Palb: Palbociclib.
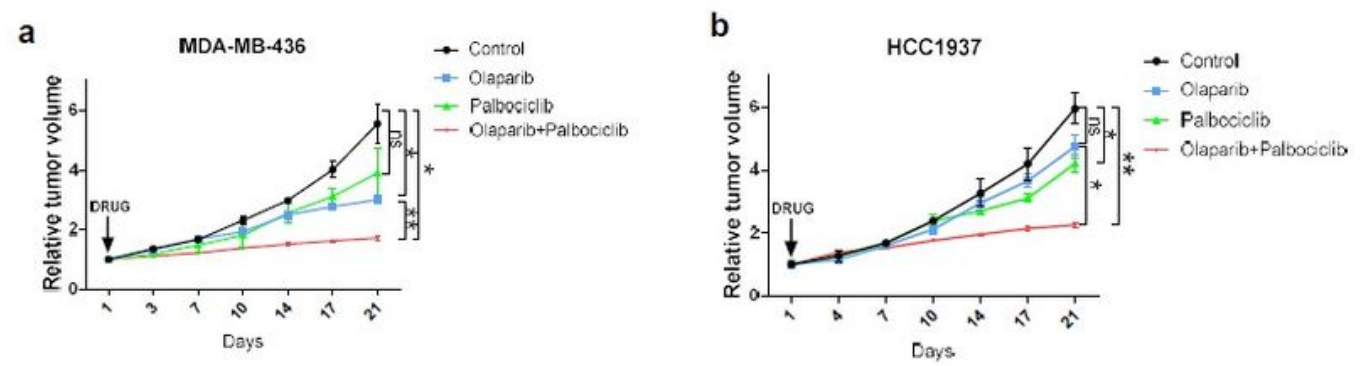

C

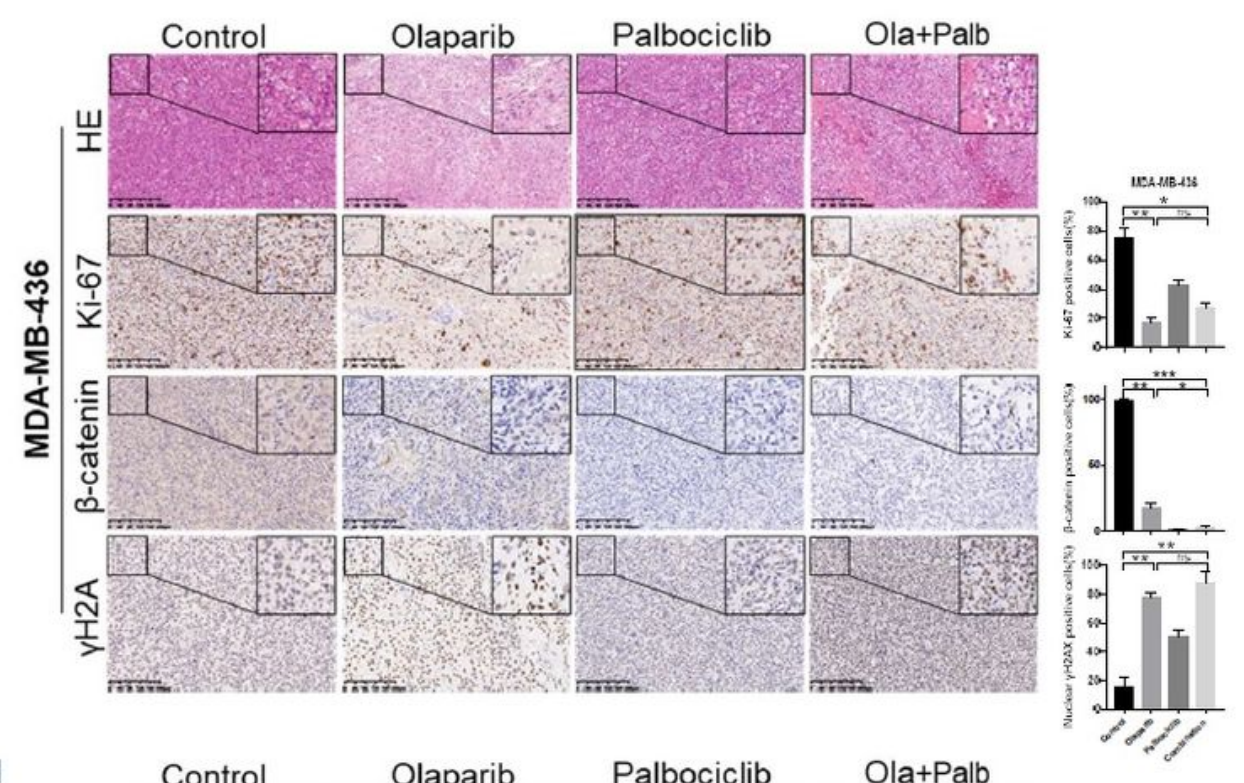

d

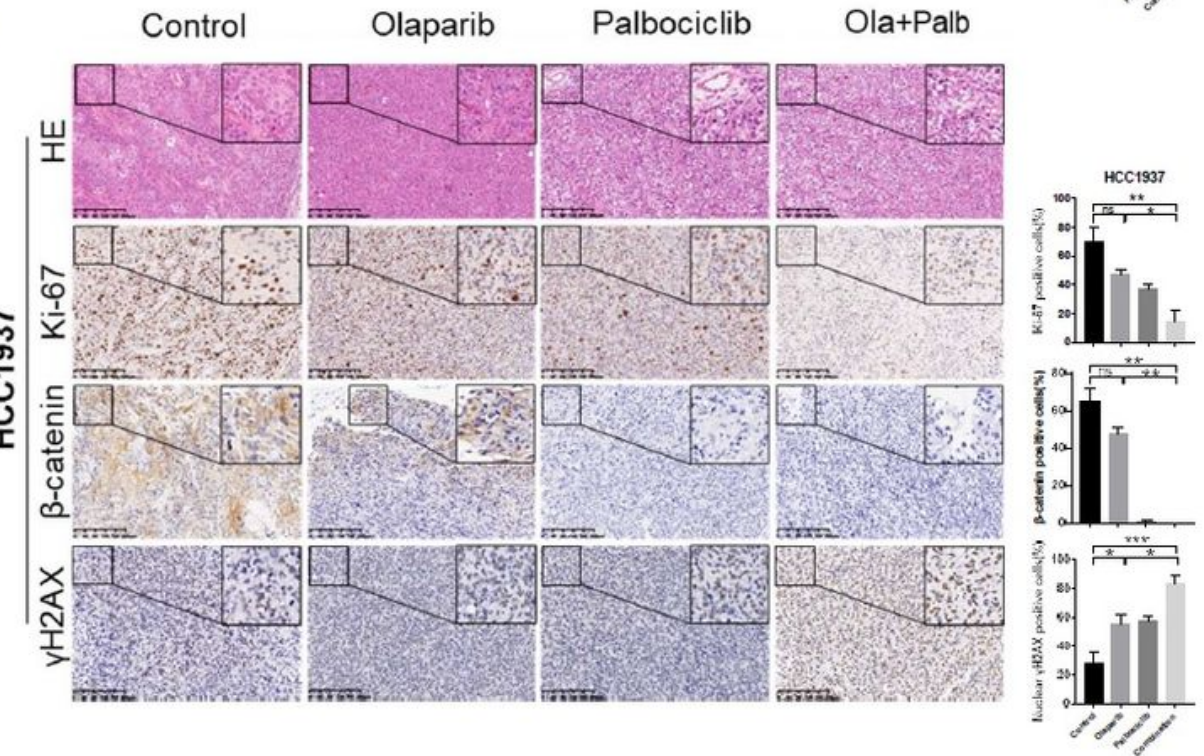

Figure 5

The combined treatment of Olaparib and Palbociclib is effective in vivo. $a$ and $b$ Tumor growth curves of (A) MDA-MB-436 and (B) HCC1937 xenografted NOD-SCID mice treated with olaparib (50 mg/kg/day) 
and palbociclib (100 mg/kg/day), either alone or in combination, for 21 days. The arrow indicates the start of treatment. $c$ and d Representative images of immunohistochemical staining as indicated in the xenografted tumors ( $\mathrm{n} \geq 3$ per treatment group) treated with olaparib and palbociclib either as single agents or in combination for 21 days. Scale bar, $200 \mu \mathrm{m}$. The small image inside is 4 times larger than the big image. Student's t-test; ${ }^{* \star} p<0.001,{ }^{\star \star} p<0.01,{ }^{\star} p<0.05$; ns, not significant. The data are presented as the means \pm SEMs. Con: Control; Ola: Olaparib; Palb: Palbociclib.

\section{Supplementary Files}

This is a list of supplementary files associated with this preprint. Click to download.

- Additionalfile1Supplementarytable.xIsx

- Additionalfile1Supplementarytable.xIsx

- Additionalfile2Supplementaryfigure.pptx

- Additionalfile2Supplementaryfigure.pptx 